\title{
Numerical modeling of cosmic rays in the heliosphere: Analysis of proton data from AMS-02 and PAMELA
}

\author{
E. Fiandrini, ${ }^{1}$ N. Tomassetti $\odot,{ }^{1}$ B. Bertucci, ${ }^{1}$ F. Donnini, ${ }^{2}$ M. Graziani, ${ }^{1}$ B. Khiali, ${ }^{3}$ and A. Reina Conde ${ }^{4}$ \\ ${ }^{1}$ Dipartimento di Fisica e Geologia, University of Perugia, Italy \\ ${ }^{2}$ INFN, Sezione di Perugia, Italy \\ ${ }^{3} I N F N$, Sezione di Roma Tor Vergata and ASI Space Science Data Center (SSDC), Roma, Italy \\ ${ }^{4}$ Instituto de Astrofsica de Canarias (IAC), Universidad de La Laguna, Tenerife, Spain
}

(Received 25 February 2021; accepted 27 May 2021; published 13 July 2021)

\begin{abstract}
Galactic cosmic rays (CRs) inside the heliosphere are affected by solar modulation. To investigate this phenomenon and its underlying physical mechanisms, we have performed a data-driven analysis of the temporal dependence of the CR proton flux over the solar cycle. The modulation effect was modeled by means of stochastic simulations of cosmic particles in the heliosphere. The model was constrained using measurements of CR protons made by AMS-02 and PAMELA experiments on a monthly basis from 2006 to 2017. With a global statistical analysis of these data, we have determined the key model parameters governing CR diffusion, its dependence on the particle rigidity, and its evolution over the solar cycle. Our results span over epochs of solar minimum and solar maximum, as well as epochs with magnetic reversal and opposite polarities. Along with the evolution of the CR transport parameters, we study their relationship with solar activity proxies and interplanetary parameters. We find that the rigidity dependence of the parallel mean free path of CR diffusion shows a remarkable time dependence, indicating a long-term variability in the interplanetary turbulence that interchanges across different regimes over the solar cycle. The evolution of the diffusion parameters shows a delayed correlation with solar activity proxies, reflecting the dynamics of the heliospheric plasma, and distinct dependencies for opposite states of magnetic polarity, reflecting the influence of charge-sign-dependent drift in the CR modulation.
\end{abstract}

DOI: $10.1103 /$ PhysRevD.104.023012

\section{INTRODUCTION}

Galactic cosmic rays (CRs) are high-energy charged particles produced by astrophysical sources, distributed in our Galaxy, which travel through the interstellar medium and finally arrive at the boundary of the nearby region to Earth, where the Sun's activity dominates: the so-called heliosphere. When entering the heliosphere, CRs travel against the expanding solar wind (SW) and interact with the turbulent heliospheric magnetic field (HMF) [1]. They are subjected to basic transport processes such as convection, diffusion, and adiabatic energy losses. They are also subjected to the gradient-curvature drifts in the large-scale HMF and to the effects of the heliospheric current sheet (HCS). Magnetic drift depends on the charge sign of the particles and on the polarity of the HMF; CRs drift along different trajectories according to the polarity of the HMF. The cumulative effects of these processes are behind the

Published by the American Physical Society under the terms of the Creative Commons Attribution 4.0 International license. Further distribution of this work must maintain attribution to the author(s) and the published article's title, journal citation, and DOI. so-called solar modulation phenomenon of CRs, that is, the modification of the energy spectra of CRs in the heliosphere, which is driven by the Sun's magnetic activity. Because of solar modulation, the CR flux observed at Earth is significantly different from that in interstellar space, known as the local interstellar spectrum (LIS). Solar modulation depends on the CR particle species, its energy, and its charge sign. It is also a time-dependent and space-dependent phenomenon; i.e., it depends on where and when the CR flux is measured inside the heliosphere. The solar modulation effect decreases with increasing energy of the CR particles. With the precision of the new CR data from Alpha Magnetic Spectrometer (AMS-02), the modulation effect is appreciable at kinetic energies up to dozens of GeV. Solar activity shows an 11-year cycle, from its minimum when the Sun is quiet and the CR intensity is at its largest to its maximum of solar activity when the CR flux is minimum. The intensity and the energy spectra of the CR flux are, therefore, anticorrelated with solar activity, in relation to its varying proxies such as the number of sunspots ( $\mathrm{SSN}$ ) or the tilt angle of the solar magnetic axis with respect to the rotation axis $\alpha$ [2-4]. Along with the 11-year solar cycle, the HMF polarity shows a remarkable 22-year periodicity, with the magnetic reversal occurring 
during each maximum of solar activity. This periodicity is important for CR modulation and, in particular, to study the effects of particle drifts in the large-scale HMF.

Since CR modulation is a manifestation of the CR propagation through the heliosphere, CR data can be used to investigate the fundamental physics processes governing the transport of charged particles through the heliospheric plasma. In particular, precise measurements of the energy and time dependence of the CR fluxes may help to disentangle the interplay of the different physics mechanisms at work. In this respect, the physical understanding of $\mathrm{CR}$ modulation in the heliosphere is one of the main objectives of many theoretical and observational studies [5-8]. Besides, modeling the CR modulation is essential for the search of new physics signatures in the fluxes of CR antimatter such as positrons or antiprotons. An antimatter excess in CRs may suggest the occurrence of dark matter annihilation processes or the existence of new astrophysical sources of antimatter. Since the low-energy spectra of CRs are influenced by solar modulation, any interpretation about the origin of antiparticles requires an accurate modeling of the charge-sign- and energy-dependent effects of CR modulation [9]. Understanding the evolution of the CR fluxes in the heliosphere is also important for assessing the radiation hazard to astronauts, electronics, and communication systems for low-Earth-orbit satellites or deepspace missions [10,11]. In fact, the Galactic CR flux constitutes a significant dose of ionizing radiation for human bodies and electronics, and, thus, an accurate knowledge of the temporal and spatial variation of the $\mathrm{CR}$ in the heliosphere will reduce the uncertainties in the radiation dose evaluation [12]. An important challenge, in this context, is to establish a predictive model for solar modulation that is able to forecast the CR flux evolution using solar activity proxies.

From the observational point of view, substantial progress has been made with the new measurements of the proton flux from the AMS-02 experiment in the International Space Station [13,14] and the PAMELA mission on board the Resurs-DK1 satellite $[15,16]$, along with the data provided by the Voyager-1 spacecraft beyond the heliosphere [17]. In particular, AMS-02 and PAMELA have recently released accurate measurements of $\mathrm{CR}$ proton spectra over Bartels' rotation (BR) basis (27 days), over an extended energy range and for extended time periods, covering the long solar minimum of 2006-2009 (cycle $23 / 24$ ), the ascending phase of cycle 24 , the solar maximum and HMF reversal of 2013-2014, and the subsequent descending phase toward the new minimum until May 2017. Therefore, the data allow for the study of the CR propagation in the heliosphere under very different conditions of solar activity and epochs of opposite HMF polarities, which may bring a substantial advance in the understanding of the solar modulation phenomenon.
In this paper, we present a data-driven analysis of the temporal dependence of the flux of CR protons, which constitute the most abundant species of Galactic cosmic radiation. The analysis has been conducted using a stochastic model of CR propagation, i.e., a Monte Carlobased approach in which the solar modulation effect is computed by statistical sampling. Using the recent time- and energy-resolved measurements of CR proton fluxes on BR basis, by means of a procedure of statistical inference, we determine the temporal and rigidity dependencies of the mean free path of CRs propagating through the heliosphere, along with the corresponding uncertainties. The rest of this paper is organized as follows. In Sec. II, we describe in detail the numerical implementation of the CR modulation model, which is based on known and conventional mechanisms of particle transport in the heliosphere. In Sec. III, we present the procedure for the datadriven determination of the key model parameters and their uncertainty, which is based on a grid sampling over a multidimensional parameter space. In Sec. IV, we present the fit results and discuss their interpretation, in terms of physical mechanisms of $\mathrm{CR}$ transport, in relation to the properties of heliospheric environment or with known proxies of solar activity. We then conclude, in Sec. V, with a summary of our study and a discussion on its future developments.

\section{THE NUMERICAL MODEL}

To get a realistic description of CR modulation phenomenon, one needs to capture the essential features of CR transport in the heliosphere. The diffusive propagation of the charged particles in the turbulent heliospheric plasma is described by the Parker equation [18]:

$\frac{\partial f}{\partial t}+\nabla \cdot\left(\vec{V}_{\mathrm{sw}}-\mathbf{K} \cdot \nabla f\right)-\frac{1}{3}\left(\nabla \cdot \vec{V}_{\mathrm{sw}}\right) \frac{\partial f}{\partial(\ln R)}=0$.

The equation, along with its boundary conditions, describes the evolution of the distribution function $f(t, \vec{r}, R)$ for a given particle species, where $t$ is the time and $R$ is the particle rigidity, i.e., the momentum per charge units $R=p / Z$. In this paper, we will focus on cosmic protons, so that $R \equiv p$. The quantity $\mathbf{K}$ is the drift-diffusion tensor of the CR particles in the turbulent HMF of the heliosphere.

Because of the complexity of the transport equation, analytical solutions can be found only for very simplified situations such as in the force-field or the diffusionconvection approximations $[19,20]$. The full solution of Eq. (1) can be obtained numerically. Here, we employ the stochastic method that has become widely implemented in recent years thanks to the enormous progress in computing speed and resources [8,21,22]. The method consists of transforming the Parker equation into a set of stochastic differential equations (SDEs) and then using Monte Carlo simulations to sample the solution, i.e., the 
differential CR intensity for a given species, at a given position in the heliosphere $[23,24]$.

In general, the flux of CRs inside the heliosphere is time dependent, reflecting the varying conditions of the medium over which they propagate [25]. A common practice is to follow a quasi-steady-state approximation where the timedependent CR modulation is described as a succession of steady-state solutions $(\partial / \partial t=0)$ and the effective status of the heliospheric plasma during the $\mathrm{CR}$ propagation is defined in a suitable way. The approximate way of taking into account the varying status of the heliosphere during the CR propagation is described in Sec. II. Furthermore, in the SDE method, pseudoparticles are propagated backward in time from the Earth position to the heliospheric boundaries. The numerical engine for handling the Monte Carlo generation and the trajectory tracing is extracted from the publicly available code SolarProp [21]. Based on the SolarProp simulation framework, we have implemented a customized model that is described in the following.

\section{A. The modulation region}

The heliosphere is a dynamic void in the interstellar medium (ISM) generated by the SW and regulated by Sun's activity. The relevant boundary for the $\mathrm{CR}$ modulation phenomenon is the heliopause (HP), which separates the heliospheric plasma from the local ISM. The HP is usually modeled as a spherical structure of radius $r_{\mathrm{HP}} \approx 122 \mathrm{AU}$, where the Sun lies at its center. Within the heliosphere, the termination shock (TS) is located at $r_{\mathrm{TS}} \cong 85 \mathrm{AU}$, while the Earth position is at $r_{0} \equiv 1 \mathrm{AU}$ placed in the equatorial plane.

\section{The large-scale $\mathrm{HMF}$}

The outward-flowing SW embeds a frozen-in HMF, which is wound up in a modified Parker spiral [26]. The ideal Parker field is given by

$$
\vec{B}=A B_{0}\left(\frac{r_{0}}{r}\right)^{2}\left(\hat{e}_{r}-\tan \psi \hat{e}_{\phi}\right)[1-2 H(\theta-\Theta)],
$$

where $r$ and $\theta$ are the helioradius and colatitude, respectively, $B_{0}$ is the HMF value at Earth position, $A= \pm 1$ is the field polarity, and $H$ is the Heaviside step function. The winding angle $\psi$ of the field line is defined as $\tan \psi=\Omega\left(r-r_{\odot}\right) \sin \theta / V_{\text {sw }}$; the angle $\Theta$ determines the position of the wavy HCS, given by $\Theta=\pi / 2+$ $\sin ^{-1}\left[\sin \alpha \sin \left(\Omega r / V_{w}\right)\right]$ [27]. Here, the quantity $\Omega$ is the average equatorial rotation speed $\approx 2.73 \times 10^{-6} \mathrm{rad} \mathrm{s}^{-1}, \alpha$ is the HCS tilt angle, and $r_{\odot}=696.000 \mathrm{~km}$ is the radius of the Sun. The Parker model overwinds by several degrees beyond the value of the winding angle $\psi$, determined by the model at the polar regions. To avoid this, one has to consider that solar wind disturbances and plasma waves propagating along the open field lines modify the magnetic field at the polar regions, so that it does not degenerate to a straight line along the polar axis. Here, we adopt the modification of Jokipii and Kota [28]:

$$
B=B_{0}\left(\frac{r_{0}}{r}\right)^{2}\left\{1+\tan ^{2} \psi+\left(\frac{r \delta(\theta)}{r_{\odot}}\right)^{2}\right\}^{1 / 2},
$$

where $\delta(\theta)=8.7 \times 10^{-5} / \sin (\theta)$ if $1.7^{\circ}<\theta<178.3^{\circ}$ and $\simeq 3 \times 10^{-3}$ otherwise [29]. The winding angle $\psi$ is then modified as

$$
\tan \psi=\left\{\frac{\Omega\left(r-r_{\odot}\right)}{V}+\left(\frac{r \delta(\theta)}{r_{\odot}}\right)^{2}\right\}^{1 / 2} .
$$

The term involving the dimensionless constant $\delta$ reflects the fact that the random field is equivalent to a small latitudinal component $B_{\theta} \sim \delta(\theta) r / r_{\odot}$. In this way, modifications on HMF and winding angle are effective only near the polar regions, as shown in Fig. 1, where the two quantities are shown as a function of colatitude. It is worth noticing that the definitions of $B_{\theta}$ and $\delta(\theta)$ imply $\vec{\nabla} \cdot \vec{B}=0$.

\section{Polarity and tilt angle}

An important characteristic for the CR solar modulation is that the HMF follows an 22 -year cycle, known as the magnetic polarity cycle, characterized by a N/S reversal about every $\sim 11$ years, during the maximum of solar activity. The period when $\vec{B}$ is directed outward in the northern hemisphere of the Sun is known as the positive polarity epoch $(A>0)$, while when it has the opposite direction as the $(A<0)$ cycle. In practice, the quantity $A$ is a dichotomous variable that expresses the sign of $B$-field projection in the outward direction from the northern
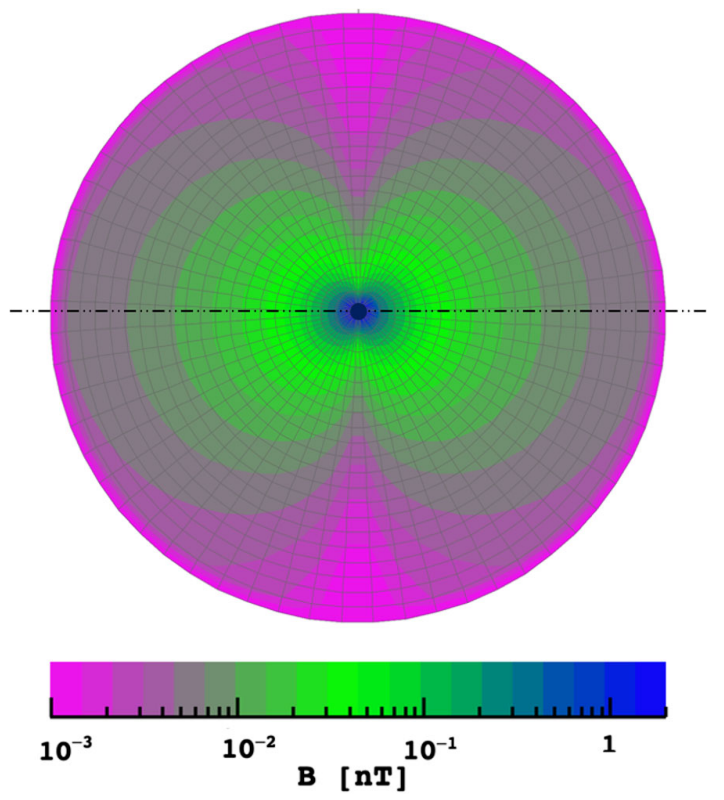

FIG. 1. Side view of the HMF field model in the $(x, z)$ plane of the heliosphere. The dashed line is the equatorial plane. 
hemisphere, $A \equiv B_{N} /\left|B_{N}\right|$ (or the inward projection of $B_{S}$ in the southern hemisphere). In practice, it can be determined using observations of the polar HMF in proximity of the Sun (Sec. III B). The relevance of magnetic polarity in the context of solar modulation arises from CR drift motion: It can be seen (Sec. II B) that the equations ruling CR drift in the HMF depend upon the sign of the product between $A$ and $\hat{q}=Q /|Q|$, where $Q$ is the CR electric charge. Thus, opposite drift directions are expected for opposite $\hat{q} A$ conditions. A major corotating structure relevant to $\mathrm{CR}$ modulation is the $\mathrm{HCS}$, which divides the HMF into hemispheres of opposite $(\mathrm{N} / \mathrm{S})$ polarity and where $B=0$. Because of the tilt of the solar magnetic axis, the HCS is wavy. The level of the HCS waviness changes with time, and it is set by the tilt angle $\alpha(t)$. Typically, it varies from $\alpha \sim 5^{\circ}$ during solar minimum to $\alpha \sim 70^{\circ}$ during solar maximum. The tilt angle is reconstructed by the Wilcox Solar Observatory using two different models for the polar magnetic field: the so-called $\mathrm{L}$ model and $\mathrm{R}$ model. In this work, the classical L-model reconstruction is used as the default.

\section{The wind}

The SW speed $V_{\text {sw }}$ is taken as radially directed outward. However, the wind field exhibits a radial, latitudinal, and temporal dependence, where the latter is related to the solar cycle. During periods of solar minimum, the flow becomes distinctively latitude dependent, changing from $\sim 400 \mathrm{~km} \mathrm{~s}^{-1}$ in the equatorial plane (slow-speed region) to $\sim 800 \mathrm{~km} \mathrm{~s}^{-1}$ in the polar regions (high-speed region), as observed by Ulysses [30]. This effect is mitigated during epochs of solar maximum, when the angular extension of the slow-speed region increases to higher latitudes. Beyond the TS, the SW slows down by a factor $1 / S$, where $S=2.5$ is the shock compression ratio, as measured by the Voyager probes [31]. In this region, the wind is slowed down to subsonic speed. To incorporate such features in our model, we adopt the parametric expression given in Ref. [32]:

$$
\begin{aligned}
V_{\mathrm{sw}}(r, \theta)= & V_{0}\left\{1.475 \mp 0.4 \tanh \left[6.8\left(\theta-\pi / 2 \pm \theta_{T}\right)\right]\right\} \\
& \times\left[\frac{S+1}{2 S}-\frac{S-1}{2 S} \tanh \left(\frac{r-r_{\mathrm{TS}}}{L}\right)\right],
\end{aligned}
$$

where $V_{0}=400 \mathrm{~km} \mathrm{~s}^{-1}$ and $L=1.2 \mathrm{AU}$ is the scale thickness of the TS. The top and bottom signs correspond to the northern $(0 \leq \theta \leq \pi / 2)$ and southern hemisphere $(\pi / 2 \leq \theta \leq \pi)$ of the heliosphere, respectively. The angle $\theta_{T}$ determines the polar angle at which the SW speed changes from a slow to a fast region. It is defined as $\theta_{T}=\alpha+\delta \alpha$, where $\alpha$ is the tilt angle of the HCS and $\delta \alpha=10^{\circ}$ is the width of the transition. With this approach, the angular extension $\theta_{T}$ of the SW profile changes in time, and it is linked to the level of solar activity, using the angle $\alpha$ as proxy. The expression is valid for $r \gg r_{\odot}$, i.e., away
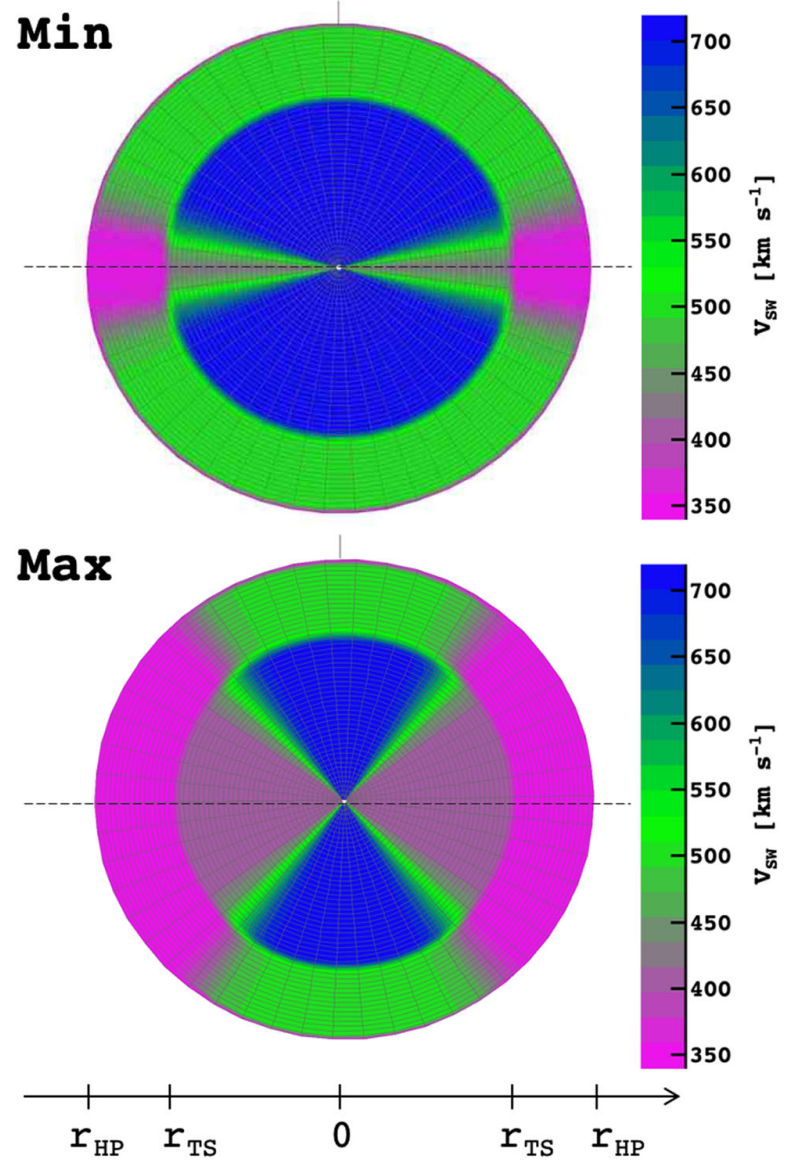

FIG. 2. Side view of the SW speed profile in the $(x, z)$ of the heliosphere, showing its latitudinal dependence in the typical cases of solar minimum (min, for $\alpha \cong 10^{\circ}$ ) and solar maximum (max, for $\alpha \cong 60^{\circ}$ ), where the latitudinal transition from a slow to a fast region depends on the HCS tilt angle $\alpha$.

from the Sun. Beyond the TS, the real SW speed is expected to decrease as $r^{-2}$, so that $\vec{\nabla} \cdot \overrightarrow{V_{\mathrm{sw}}}=0$ and CR particles do not experience adiabatic cooling. The radial and latitudinal SW profile is shown in Fig. 2 for two values of $\alpha$ corresponding to solar minimum $\left(\alpha \cong 10^{\circ}\right)$ and solar maximum $\left(\alpha \cong 60^{\circ}\right)$ conditions.

\section{B. The particle transport}

The Parker equation for the particle transport contains all physical processes experienced by a given species of CR particles traveling in the interplanetary space. In Eq. (1), the drift-diffusion tensor can be written as

$$
\mathbf{K}=\left[\begin{array}{ccc}
K_{r \perp} & -K_{A} & 0 \\
K_{A} & K_{\theta \perp} & 0 \\
0 & 0 & K_{\|}
\end{array}\right]
$$

in a reference system with the third coordinate along the average magnetic field. The symbol $K_{\|}$denotes the diffusion coefficient along the field direction, while $K_{\theta \perp}$ and $K_{r \perp}$ are 
the diffusion coefficients along the perpendicular and radial direction, respectively. $K_{A}$ expresses the value of the antisymmetric part of the diffusion tensor, where its explicit form results from the effects on the motion of CR particles due to drift. $\vec{V}_{\mathrm{sw}}$ is the $\mathrm{SW}$ speed, and $\vec{V}_{D}$ is the guiding center speed for a pitch-angle-averaged nearly isotropic distribution function. The equation can be then rewritten as

$$
\begin{gathered}
\frac{\partial f}{\partial t}-\nabla \cdot\left[\mathbf{K}^{S} \cdot \nabla f\right]+\left(\vec{V}_{\mathrm{sw}}+\vec{V}_{D}\right) \cdot \nabla f \\
-\frac{\left(\nabla \cdot \vec{V}_{\mathrm{sw}}\right)}{3} \frac{\partial f}{\partial(\ln R)}=0 .
\end{gathered}
$$

The motion of the CR particles in the HMF is usually decomposed in a regular gradient-curvature and HCS drift motion on the background average HMF and a diffusion due to the random motion on the small-scale fluctuations of the turbulent HMF. All these effects are included in the diffusion tensor $\mathbf{K}$ of Eq. (6), which can be decomposed in a symmetric part that describes the diffusion and an antisymmetric one that describes the drifts, i.e., $\mathbf{K}=\mathbf{K}^{S}+\mathbf{K}^{A}$, with $K_{i j}^{S}=K_{j i}^{S}$ and $K_{i j}^{A}=-K_{j i}^{A}$. Particles moving in a magnetic turbulence are pitch-angle scattered by the random HMF irregularities. This process is captured by the symmetric part of the diffusion tensor $\mathbf{K}^{S}$, which is diagonal if the $z$ coordinate is aligned with the background HMF. Three diffusion coefficients are therefore needed, namely, parallel diffusion $K_{\|}$, transverse radial $K_{\perp r}$, and transverse polar diffusion coefficient $K_{\perp \theta}$. The coefficients can also be expressed in terms of mean free path $\lambda$ along the background HMF, e.g., $K_{\|}=\beta c \lambda_{\|} / 3$ (with $\beta=v / c$ ). The determination of the diffusion coefficients is a key ingredient to study the propagation of charged particles in turbulent magnetic fields like the HMF and is the subject of many theoretical and computational studies. The quasilinear theory (QLT) has been successful at describing parallel diffusion, especially in its time-dependent and nonlinear extensions [33]. Regarding perpendicular diffusion, the QLT provides upper limits within the field line random walk description [33,34], while the best approaches follow the nonlinear guiding center theory [35-37].

From a microscopic point of view, CR diffusion is linked to the resonant scattering of particles with rigidity $R$ with the HMF irregularities around the wave number $k_{\text {res }} \sim$ $2 \pi / r_{L}$, where $r_{L}=R / B$. The essential dependence of $\lambda_{\|}$ on the HMF power spectrum can be expressed as $\lambda_{\|} \sim r_{L}^{2}\left\langle B^{2}\right\rangle / w\left(k_{\text {res }}\right) \sim R^{2} / w\left(k_{\text {res }}\right)$, where $\left\langle B^{2}\right\rangle$ is the mean square value of the background field and $w\left(k_{\text {res }}\right)$ is the power spectrum of the random fluctuations of the HMF around the resonant wave number. The power spectral density follows a power law as $w(k) \sim k^{-\nu}$, where the index $\nu$ depends on the type and on the spatial scales of the turbulence energy cascade $[38,39]$. Therefore, $\lambda_{\|}$depends on the turbulence spectral index as $\lambda_{\|} \sim R^{2-\nu}$. In this work, for the rigidity and spatial dependence of the parallel diffusion coefficient, we adopt a double power-law rigidity dependence and an inverse proportionality with the local HMF magnitude, following Ref. [32]:

$$
K_{\|}=K_{0} \frac{\beta}{3} \frac{\left(R / R_{0}\right)^{a}}{\left(B / B_{0}\right)}\left[\frac{\left(R / R_{0}\right)^{h}+\left(R_{k} / R_{0}\right)^{h}}{1+\left(R_{k} / R_{0}\right)^{h}}\right]^{\frac{b-a}{h}} .
$$

In this expression, $K_{0}$ is a constant of the order of $10^{23} \mathrm{~cm}^{2} \mathrm{~s}^{-1}, R_{0}=1 \mathrm{GV}$ to set the rigidity units, $B$ the HMF magnitude, and $B_{0}$ the field value at Earth and written in a way such that the units are in $K_{0}$. Here, $a$ and $b$ are power indices that determine the slope of the rigidity dependence, respectively, below and above a rigidity $R_{k}$, whereas $h$ determines the smoothness of the transition. The perpendicular diffusion in the radial direction is calculated as $K_{\perp r}=\xi_{\perp r} \times K_{\|}$, while the polar perpendicular diffusion was parameterized as $K_{\perp \theta}=\xi_{\perp \theta} \times g(\theta) \times K_{\|}$, where $g(\theta)$ is a function that enhances $K_{\perp \theta}$ by a factor $d$ near the poles, defined as [32]

$$
g(\theta)=A^{+} \mp A^{-} \tanh \left[8\left(\theta_{A}+\pi / 2 \pm \theta_{F}\right)\right] .
$$

Here, $A^{ \pm}=(d \pm 1) / 2, \theta_{F}=35^{\circ}$, and $\theta_{A}=\theta$ if $\theta \leq \pi / 2$ or $\theta_{A}=\pi-\theta$ if $\theta \geq \pi / 2$, with $d=3$. The enhancement in the latitude direction of $K_{\perp \theta}$, together with the anisotropy between the perpendicular diffusion coefficients and HMF modification at the polar regions, is needed to account for the very small latitudinal dependence of the CR intensity, as it was observed in the Ulysses data [30,40]. The adoption of constant $\xi_{\perp}$ factors implies that $K_{\perp}$ and $K_{\|}$follow the same rigidity dependence, which may be a simplification in the high- $R$ domain [36,41]. Nonetheless, QLT-based simulations agree for nearly rigidity-independent $\xi$, with the typical value of $0.02-0.04[34,42]$. In this work, the parameters $\xi_{\perp r}$ and $\xi_{\perp \theta}$ are fixed to the value 0.02 . We now turn on drift effects, that account for the charge-sign and polarity dependence of CR transport in the HMF [27,43]. The regular motion of CRs on the large-scale HMF is given by the pitch-angle-averaged guiding center drift speed $\left\langle\vec{V}_{D}\right\rangle$. It can be related to the antisymmetric part of the diffusion tensor [44]:

$$
\left\langle\left(V_{D}\right)_{i}\right\rangle=\frac{\partial K_{i j}^{A}}{\partial x_{j}},
$$

where the antisymmetric part of the tensor has the form

$$
K_{i j}^{A}=K_{A} u(\theta) \zeta(R) \epsilon_{i j k} \frac{B_{k}}{B} .
$$

Here, $\epsilon_{i j k}$ is the Levi-Civita symbol, $u(\theta)$ is a function that describes the transition between the region influenced by the HCS and the regions outside of it, and $\zeta(R)$ is a function of rigidity that suppresses drifts at low rigidity. To determine the value of $K_{A}$, we note that the small value of the 
ratio $K_{\perp} / K_{\|}$suggests that $\mathrm{CR}$ particles move over many gyro-orbits in a mean free path; therefore, the drift motion is weakly affected by scattering. In the weak scattering approximation, one has

$$
K_{A}=K_{A}^{0} \frac{Q}{|Q|} \frac{\beta R}{3 B},
$$

where $Q$ is the CR particle charge and $K_{A}^{0}$ is a normalization factor $\leq 1$. Drift motion is relevant close the HCS, where CRs cross many times regions of opposite HMF polarity. A 2D description of HCS drift is given in Burger and Hattingh [44]. In this approach, the drift velocity is given by

$$
\left\langle\vec{V}_{D}\right\rangle=\zeta(R)[\vec{G}+\vec{H}],
$$

where the two vectors are defined as follows:

$$
\begin{aligned}
& \vec{G}=u(\theta) \nabla \times\left(K_{A} \frac{\vec{B}}{B}\right), \\
& \vec{H}=\left(\frac{\partial u(\theta)}{\partial \theta}\right)\left(\frac{K_{A}}{r}\right) \overrightarrow{e_{\theta}} \times \frac{\vec{B}}{B} .
\end{aligned}
$$

The $\vec{G}$ term in Eq. (14) describes the gradient-curvature drifts, the $\vec{H}$ term describes the particle motion across the region affected by the HCS, $\vec{e}_{\theta}$ is the unit vector along the polar direction, and $u(\theta)$ is given by

$$
u(\theta)= \begin{cases}\left(1 / a_{h}\right) \arctan \left\{\left[1-(2 \theta / \pi) \tan a_{h}\right]\right\} & \text { if } c_{h}<\pi / 2, \\ 1-2 H(\theta-\pi / 2) & \text { if } c_{h}=\pi / 2\end{cases}
$$

with $H$ the Heaviside step function,

$$
a_{h}=\arccos \left(\frac{\pi}{2 c_{h}}-1\right)
$$

and

$$
c_{h}=\frac{\pi}{2}-\frac{1}{2} \sin \left(\alpha+\frac{2 r_{L}}{r}\right) .
$$

The angle $2 r_{L} / r$ depends on the maximum distance that a particle can be away from the HCS while drifting. Finally, the function $u(\theta)$ is such that $u(\pi / 2)=0, u\left(c_{h}\right)=0.5$, and $\partial u(\pi / 2) / \partial \theta=1$. CR drift coefficients are expected to be reduced in the presence of turbulence as results theoretically and from numerical test-particle simulations [45,46]. In this work, we use a simple approach to incorporate drift reduction. Following Ref. [46], we adopt a reduction factor of the type

$$
\zeta=\frac{1}{1+\frac{R_{A}^{2}}{R^{2}}}
$$

where the reduction occurs at rigidity below the cutoff value $R_{A}=\lambda_{\perp} \delta B_{T}$, which depends on the perpendicular diffusion length and total variance of the HMF. The reduction is effective at $R \ll R_{A}$, when $\zeta \approx\left(R / R_{A}\right)^{2} \ll 1$, while in the high- $R$ limit one has $\zeta \approx 1$. The cutoff value $R_{A}$ depends on the HMF turbulence through $\lambda_{\perp}$ and $\delta B_{T}$. With typical values of $\lambda_{\perp} \approx 1.5 \times 10^{-3} \mathrm{AU}$ and $\delta B_{T} \approx$ $3.5 \mathrm{nT}$ for the considered epochs, one can estimate $R_{A} \approx 0.3-0.6 \mathrm{GV}$. In this work, we have fixed it at $0.5 \mathrm{GV}$, corresponding to a proton kinetic energy of $125 \mathrm{MeV}$. The normalization $K_{A}^{0}$ factor is fixed to 1 , so that the whole drift reduction is regulated by $\zeta$.

The most relevant feature of magnetic drift is that its direction depends on the sign of the charge, $\hat{q}=Q /|Q|$, and on the HMF polarity $A$, via the product $\hat{q} A$, so that particles with opposite $\hat{q} A$ will drift in opposite directions and will follow different trajectories in the heliosphere. This characteristic is expected to give observable chargesign dependence in the CR modulation. Finally, in a reference frame with the $z$ coordinate along the average magnetic field, the diffusion tensor is given by Eq. (6). The effective diffusion tensor in heliocentric polar coordinates is obtained by a coordinate transformation in the modified Parker field. In our 2D approach, the relevant components are $K_{r r}=K_{\|} \cos ^{2} \psi+K_{\perp r} \sin ^{2} \psi$, $K_{\theta \theta}=K_{\perp \theta}$, and $K_{\theta r}=K_{A} \sin \psi=-K_{r \theta}$.

\section{The proton LIS}

To resolve the modulation equation for cosmic protons, their LIS must be specified as a boundary condition. The determination of the CR proton LIS requires a dedicated modeling effort, starting from the distribution of Galactic CR sources and accounting for all the relevant physical processes that occur in the interstellar medium. In this work, we adopt an input LIS for CR protons that relies on a two-halo model of CR propagation in the Galaxy $[47,48]$. In this model, the injection of primary CRs in the ISM is described by rigidity-dependent source terms $S \propto$ $(R / \mathrm{GV})^{-\gamma}$ with $\gamma=2.28 \pm 0.12$ for protons. The diffusive transport in the $L$-sized Galactic halo is described by an effective diffusion coefficient $D=\beta D_{0}(R / G V)^{\delta_{i / o}}$ with $D_{0} / L=0.01 \pm 0.002 \mathrm{kpc} / \operatorname{Myr}[9,48]$. The two spectral indices $\delta_{i / o}$ describe two different diffusion regimes in the inner and outer halo, with $\delta_{i}=0.18 \pm 0.05$ for $|z|<\xi L$ (inner halo) and $\delta_{o}=\delta_{i}+\Delta$ for $|z|>\xi L$ (outer halo), with $\Delta=0.55 \pm 0.11$. The $z$ variable here is the vertical spatial coordinate. The half thickness of the halo is $L \cong 5 \mathrm{kpc}$, and the near-disk region (inner halo) is set by $\xi=0.12 \pm 0.03$. Finally, we considered the impact of diffusive reacceleration. Within the two-halo model, the interstellar Alfvénic speed is constrained from the data to lie between 0 and $6 \mathrm{~km} \mathrm{~s}^{-1}$. Calculations of the proton LIS were constrained by various sets of measurements: low-energy proton data (at 140-320 MeV) collected by Voyager-1 beyond the HP 


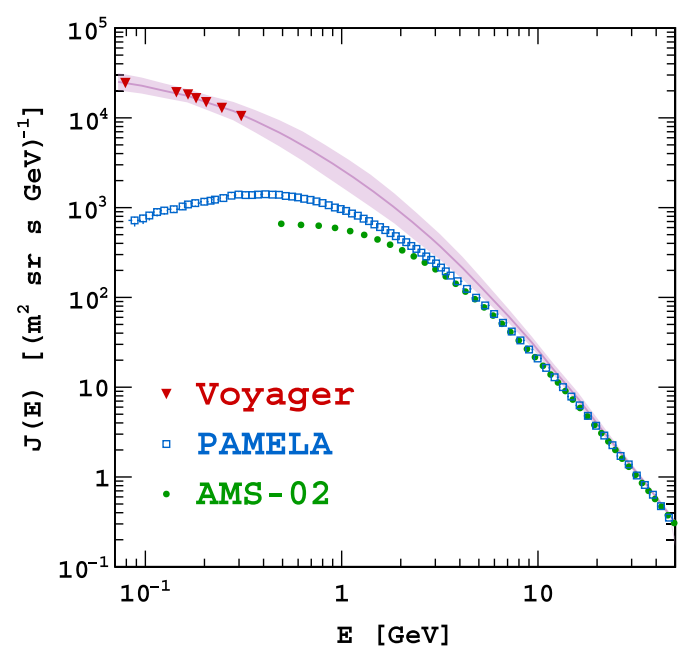

FIG. 3. Proton LIS used as input boundary condition for the modulation along with the estimated uncertainty band [47-49,53]. Data from Voyager-1 in interstellar space and from AMS-02 and PAMELA in low Earth orbit collected during two epochs.

and high-energy proton measurements $(E \gtrsim 60 \mathrm{GeV})$ made by AMS-02 in low Earth orbit, along with measurements of the $\mathrm{B} / \mathrm{C}$ ratio from both experiments. The latter were essential to constrain the diffusion parameters of the LIS model [9]. Details on this model are provided elsewhere $[48,49]$. The resulting proton LIS is shown in Fig. 3 in comparison with the data from Voyager-1, along with PAMELA and AMS-02 measurements made in March 2009 and April 2014, respectively. The uncertainty band associated with the calculations is also shown in the figure. This model is in good agreement with other recently proposed LIS models [5,22,50-52].

\section{DATA ANALYSIS}

In this section, we present the analysis method by which we extract knowledge and insights from the data using the mathematical framework described Sec. II. In practice, we defined a set of physics observables, to be computed as model predictions, and a set of model parameters, to be determined by statistical inference.

\section{A. The cosmic ray data}

The data used in this work consist in time-resolved and energy-resolved measurements of $\mathrm{CR}$ proton fluxes, in the kinetic energy range from $\sim 80 \mathrm{MeV}$ to $\sim 60 \mathrm{GeV}$. Specifically, we use the 79 BR-averaged fluxes measured by the AMS-02 experiment in the International Space Station from May 2011 to May 2017 [13] and the $47+36$ BR-averaged fluxes observed by the PAMELA instrument in the satellite Resurs-DK1 from June 2006 to January 2014 $[15,16]$. The data sample corresponds to a total of 10101 data points collected over a time range of about 11 years, from the solar minimum from 2006 to 2009, the ascending phase to solar maximum, when the HMF polarity $A$ reversed from $A<0$ to $A>0$, and the following descending phase until May 2017. These data have been retrieved by the ASI-SSDC cosmic ray database [54].

The intensity of the $\mathrm{CR}$ proton fluxes in the energy range between 0.49 and $0.62 \mathrm{GeV}$ are shown in Fig. 4 as a function of time for both the PAMELA and AMS-02 datasets. From the figure, the complementarity of the two experiments is apparent. It can be seen that the highest intensity of the CR is reached during December 2009, i.e., under the solar minimum, while the lowest intensity occurs in $\sim$ February 2014, around solar maximum. The vertical dashed line of the figure shows the HMF reversal epoch $T_{\text {rev }}$, along with the transition region shown as a shaded area where the HMF is disorganized and the polarity is not defined. The determination of $T_{\text {rev }}$ and the transition region are presented later on.

\section{B. The parameters}

The numerical model presented in Sec. II makes use of several physics input to be determined with the help of

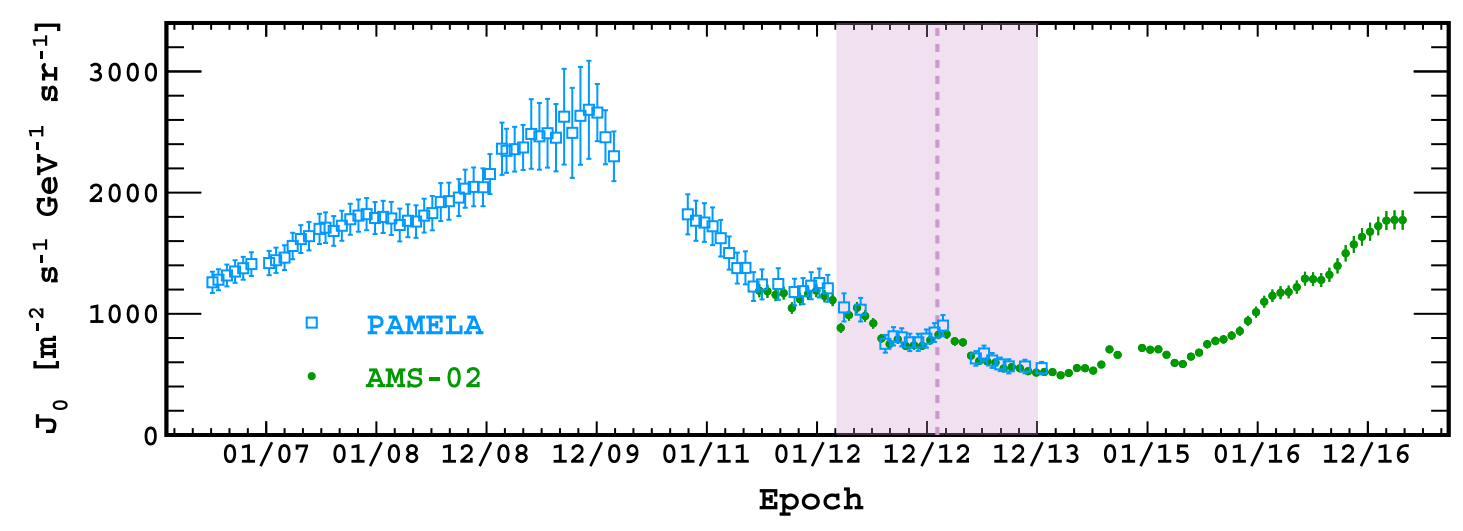

FIG. 4. BR-averaged flux $J_{0}$ evaluated in the reference energy range between 0.49 and 0.62 GeV from PAMELA (open squares) $[15,16]$ and AMS-02 (filled circles) $[13,14]$. The vertical dashed line shows the epoch of the HMF polarity inversion, along with the shaded area indicating the reversal epoch. 
observations. Inputs include solar parameters, characterizing the conditions of the Sun or the interplanetary plasma, and transport parameters that describe the physical mechanisms of CR propagation through the plasma. Solar and transport parameters are interconnected with each other, and they may show temporal variations related to the solar cycle. For instance, solar parameters such the magnetic field magnitude, its variance, and its polarity are transported from the Sun into the outer heliosphere, therefore provoking time-dependent $\mathrm{CR}$ diffusion and drift.

We identified, in our model, a set of six time-dependent key parameters that are of relevance for the phenomenology of CR modulation. They are the tilt angle of the HCS $\alpha(t)$, the strength of the HMF at the Earth location $B_{0}(t)$, the HMF polarity $A(t)$, and the three diffusion parameters appearing in Eq. (8): the normalization factor of the parallel diffusion tensor, $K_{0}(t)$, and the two spectral indices of the rigidity dependence of CR diffusion, $a(t)$ and $b(t)$, below and above the break $R_{k}$, respectively, as seen in Eq. (8). Note that all key parameters are expressed as continuous functions of time $t$, but, in practice, they have been determined for the epochs corresponding to the CR flux measurements.

The three solar parameters $\alpha, B_{0}$, and $A$ can be determined from solar observatories: Data of HMF polarity and tilt are provided by the Wilcox Solar Observatory on 10-day or BR basis. Measurements of the $\mathrm{HMF} B_{0}$ at $1 \mathrm{AU}$ are done in situ on a daily basis, since 1997, by the Advanced Composition Explorer (ACE) on a Lissajous orbit around $L 1$ [55]. It is important to notice that, in this study, our aim is to capture the effective status of the largescale heliosphere sampled by CRs detected at a given epoch $t$, and this is connected to solar-activity parameters that are precedent to that epoch. In fact, several studies have reported a time lag of a few months between the solar activity and the varying CR fluxes [51,56], reflecting the fact that the perturbations induced by the Sun's magnetic activity take a finite amount of time to establish their effect in the heliosphere. To tackle this issue, for each epoch $t$ associated to a given CR flux measurement, we perform a backward-moving average (BMA) for $\alpha$ and $B_{0}$, and $A$, i.e., a time average of these quantities calculated over a time window $[t-\tau, t]$. The window extent $\tau$ is the time needed by the SW plasma to transport the magnetic perturbations from the Sun to the HP boundary, which ranges between $\sim 8$ (fast SW speed) and $\sim 16$ months (slow SW speed). In the case of $\alpha$, the window is large, because the HCS is always mostly confined in the slow (equatorial) SW region. In the case of $B_{0}$, the BMA has to be computed by an integration over the latitudinal profile of the SW speed at a given epoch. Our estimations are consistent with the lag reported in other studies [51,56] and supported by correlative analysis that we made a posteriori. Figure 5 shows the reference parameters $\hat{B}_{0}$ and $\hat{\alpha}$ calculated for each reference epoch $t$ corresponding to a BR-averaged CR measurement.
A similar estimate is done for the polar magnetic field and for the resulting polarity $\hat{A}$, in Fig. 5(d). The latter can be regarded as a "smoothed" definition for the magnetic polarity $A$, otherwise dichotomous $(A= \pm 1)$. When the $\mathrm{HMF}$ is in a defined polarity state, one has $\hat{A}= \pm 1$. During the HMF reversal transition epoch (shaded area in the figures), as the polarity is not well defined, the estimate of $\hat{A}$ takes a floating value between -1 and +1 .

At this point, we also recall that several parameters entering the model that have been kept constant in the simulation, i.e., assumed to be known or time independent. The HP and TS positions were fixed at $r_{\mathrm{HP}}=122 \mathrm{AU}$ and $r_{\mathrm{TS}}=85 \mathrm{AU}$, respectively, deduced from the Voyager -1 observations. The data suggest that the TS may vary over the solar cycle of the order of a few AU, but its impact in the CR fluxes is not negligible [51].

The $h$ parameter of Eq. (8), describing the smoothness of the transition between the two diffusion regimes below and above $R_{k}$, was kept constant at $h=3$. Within the precision of the data, the $h$ parameter has no appreciable impact on the CR fluxes. Similarly, the rigidity break $R_{k}$ for $K_{\|}$was kept fixed at the value $3 \mathrm{GV}$. This parameter represents the scale rigidity value where the CR Larmor radius matches the correlation length of the HMF power spectrum, which is at the GV scale. Regarding the value of $R_{k}$, we found that time variations on this quantity do not give appreciable variations in the CR fluxes (see, e.g., Ref. [32]). The $\xi_{\perp i}$ coefficients for the diffusion tensor, for the values used here, represent a widely used assumption (e.g., Ref. [40]). The polar enhancement factor of Eq. (9) is kept constant at $d=3$ for $\xi_{\perp \theta}$ so that the condition $K_{\perp} / K_{\|} \ll 1$ is still fulfilled at the polar regions. Regarding magnetic drift, the critical rigidity $R_{A}$ of Eq. (18) is kept constant at $0.5 \mathrm{GV}$ following previous studies and independent observations on the CR latitudinal gradient $[32,57]$. This choice could be tested only with low-rigidity $\mathrm{CR}$ data $\left(R \ll R_{A}\right)$, as our results are insensitive to the exact value of $R_{A}$. The normalization factor for drifts speeds $K_{A}^{0}$ was chosen to be unity such to set "full drift" speeds in the propagation model for all the periods, and this drift reduction is entirely given by Eq. (18). Reductions in the $K_{A}^{0}$ value may occur during periods of strong magnetic turbulence, e.g., during solar maximum [25,57].

\section{The statistical inference}

\section{The parameter grid}

The transport parameters $K_{0}(t), a(t)$, and $b(t)$ have been determined from the AMS-02 and PAMELA data by means of a global fitting procedure. For this purpose, a sixdimensional discrete grid of the model parameter vector $\vec{q}=\left(\alpha, B_{0}, A, K_{0}, a, b\right)$ was built; i.e., the model was run for every node of the grid such as to produce a theoretical calculation for the $\mathrm{CR}$ proton flux. In the grid, the 


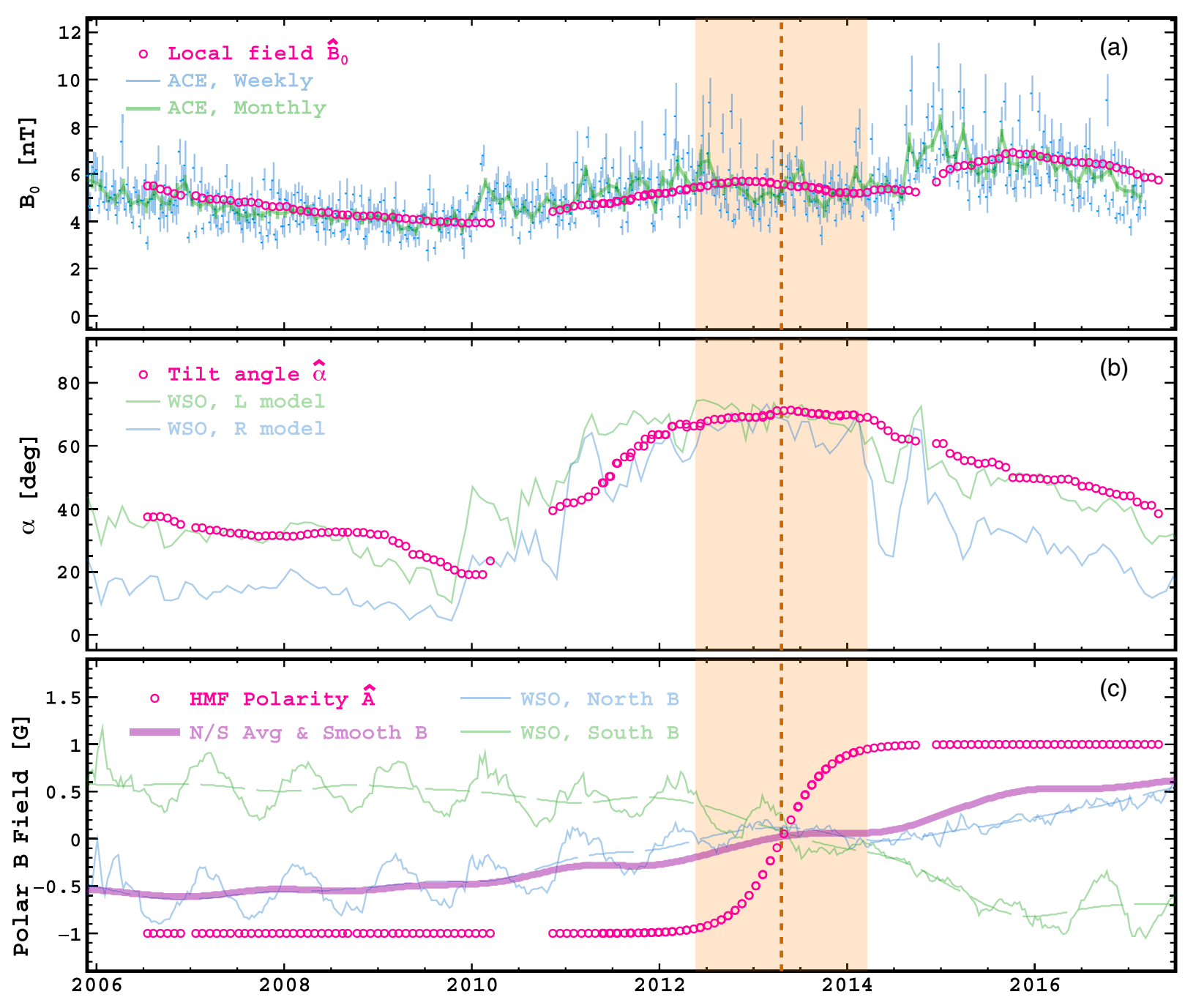

FIG. 5. Reconstruction of the tilt angle $\alpha$, the local HMF strength $B_{0}$, and the magnetic polarity $A$ as a function of the epoch, evaluated with the BMA procedure in correspondence of the epochs of the PAMELA and AMS-02 flux measurements. The vertical dashed line marks the HMF reversal $T_{\text {rev }}$. The shaded area around $T_{\text {rev }}$ represents the effective period of the HMF polarity transition. The raw data, shown as thin shaded lines, are taken from the ACE space probe and from the Wilcox Solar Observatory [4,55].

parameter $\alpha$ ranges from $5^{\circ}$ to $75^{\circ}$ with steps of $10^{\circ}$ and $B_{0}$ from 3 to $8 \mathrm{nT}$ with steps of $1 \mathrm{nT}$, and the polarity $A$ takes the two values $A=+1$ and $A=-1$. The parameter $K_{0}$ ranges from 0.16 to $1.5 \times 10^{23} \mathrm{~cm}^{2} \mathrm{~s}^{-1}$, with steps of $0.08 \times 10^{23} \mathrm{~cm}^{2} \mathrm{~s}^{-1}$, and the indices $a$ and $b$ range from 0.45 to 1.65 with steps of 0.05 . The total number of grid nodes amounts to 938400 . For each node of the parameter grid, a theoretical prediction for the modulated proton flux $J_{m}(E, \vec{q})$ was evaluated, as a function of kinetic energy, over 120 energy bins ranging from $20 \mathrm{MeV}$ to $200 \mathrm{GeV}$ with log-uniform steps. Using the SDE technique, $2 \times 10^{3}$ pseudoparticles were Monte Carlo generated and retropropagated for each energy bin. This task required the simulation of about 14 billion trajectories of pseudoprotons, corresponding to several months of CPU time. Once the full grid was completed, the output flux was tabulated and properly interfaced with the data. For each dataset
$J_{d}(E, t)$, representing a set flux measurements as a function of energy for a given epoch $t$, a $\chi^{2}$ estimator was evaluated as

$$
\chi^{2}(\vec{q})=\sum_{i} \frac{\left[J_{d}\left(E_{i}, t\right)-J_{m}\left(E_{i}, \vec{q}\right)\right]^{2}}{\sigma^{2}\left(E_{i}, t\right)} .
$$

Similarly to $J_{m}$, the $\chi^{2}$ estimator is built such as to be a continuous function of the parameters $\vec{q}$, except for the variable $A$ that is treated as discrete. From the $\chi^{2}$ estimator, the transport parameters $\left\{K_{0}, a, b\right\}$ can be determined by minimization at any epoch, while the solar parameters $\left\{B_{0}, \alpha, A\right\}$ can be considered as "fixed inputs," as they are determined by the epoch $t$ using the BMA reconstruction presented above. For a given set of BMA inputs such as $\hat{B}_{0}$ and $\hat{\alpha}$, the flux $J_{m}(E, \vec{q})$ can be expressed as a continuous 
function of the parameters by means of a multilinear interpolation over the grid nodes. In the $\alpha-B_{0}$ plane, one has $\alpha_{j}<\hat{\alpha}(t)<\alpha_{j+1}$ and $B_{0 k}<\hat{B}_{0}(t)<B_{0, k+1}$, where $\alpha_{j}$ and $B_{0, k}$ are the closest values of the grid corresponding to their BMA averages. Regarding polarity $A$, both \pm 1 evaluations were done under the assumption that the polarity is known. The flux model dependence upon energy should also be handled. In Eq. (19), $E_{i}$ are the mean measured energies reported from the experiments (coming from binned histograms). In general, the $E_{i}$ array does not correspond to the energy grid of the model. The model evaluation of $J_{m}(E, \vec{q})$ at the energy $E_{i}$ was done by $\log$ linear interpolation.

\section{The uncertainties}

The $\sigma$ factors appearing in Eq. (19) represent the total uncertainties associated with the flux. They can be written as $\sigma^{2}\left(E_{i}, t\right)=\sigma_{d}^{2}\left(E_{i}, t\right)+\sigma_{m}^{2}\left(E_{i}, t\right)$. Here, $\sigma_{d}^{2}\left(E_{i}, t\right)$ are the experimental errors associated to the flux measurement of the $i$ th energy bin around $E_{i}$, while $\sigma_{m}^{2}\left(E_{i}, t\right)$ are the theoretical uncertainties of the flux calculations evaluated at the same value of energy. Uncertainties in experimental data are of the order of $10 \%$ in the PAMELA data and 2\% in the AMS-02 data, although they depend on kinetic energy. Theoretical uncertainties include statistical fluctuations of the finite SDE generation of pseudoparticle trajectories. Uncertainties are relevant at low energy where, due to the heavy adiabatic energy losses, the Monte Carlo sampling suffers from a smaller statistics. Thus, after repeating many times the simulation with the same modulation parameters, the modulated flux will fluctuate around an average value because of the random process of pseudoparticle propagation with the SDE approach. These fluctuations can be arbitrarily reduced with the increase of the pseudoparticle generation, but at the expense of a large CPU time. The evaluation of these uncertainties can be done as follows. Given $N_{m}$ as the number of pseudoparticles that reach the boundary with energy $E$, and $N_{G}$ as the number of pseudoparticles generated at the same energy, the ratio of the modulated flux to the LIS flux is $J_{m} / J_{\text {LIS }} \approx N_{m} / N_{G}$. Since the propagation process is stochastic in nature, the relative error of the modulated flux scales as $\delta J_{m} / J_{m}=1 / \sqrt{N_{m}}$, where $N_{m}=N_{G}\left(J_{m} / J_{\text {LIS }}\right)$. We found that the generation of $N \cong 2 \times 10^{3}$ pseudoparticles for each energy bin is sufficient for being not dominated by SDE-related uncertainties. The relative uncertainties as a function of kinetic energy are shown in Fig. 6. The errors are about $\sim 10 \%-20 \%$ at $20 \mathrm{MeV}$ of energy and decrease with increasing energy. They become constant at $\sim 2 \%$ above a few GeV. A minor source of systematic error comes from the multilinear interpolation of the parameter and energy grid, i.e., from the method we used to evaluate the flux at any arbitrary set of parameters and energy. From dedicated runs, we have

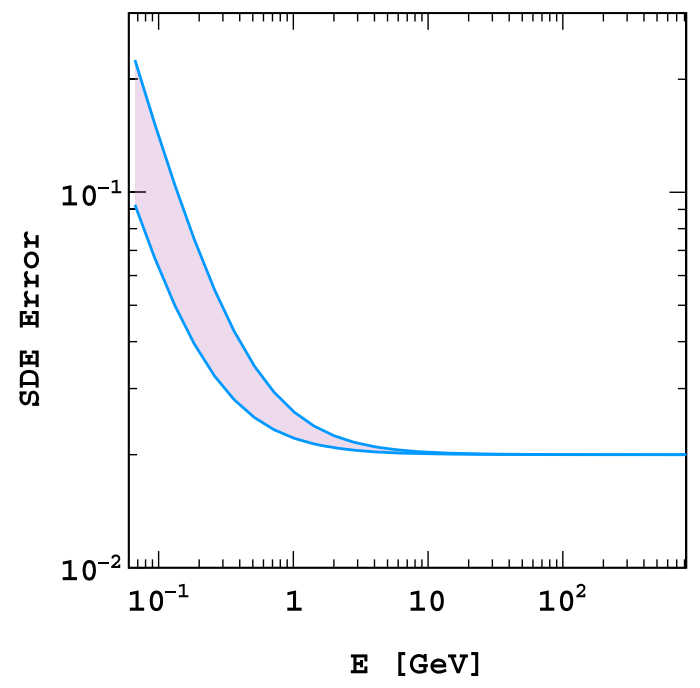

FIG. 6. Error band reflecting the statistical uncertainty of the Monte Carlo-generated trajectories. Low-energy CRs have less chance to reach the inner heliosphere, giving a higher uncertainty on the Monte Carlo statistics.

estimated that the uncertainty introduced by the interpolation, rather than the direct simulation with of $J(\vec{q}, E)$, is always of the order of $1 \%$. An important source of systematic error is the uncertainty coming from the input LIS of CR protons; see Sec. II C. The LIS uncertainties are highly energy dependent. They are significant in the energy region of $\sim 1-10 \mathrm{GeV}$ (up to $30 \%$ and more), where direct interstellar data are not available but the modulation effect is still considerable. However, in this energy region, the Galactic transport parameters regulating the LIS intensity are in degeneracy with the free parameters of CR diffusion (Sec. III B) and, in particular, with $K_{0}$ [49]. Such a degeneracy translates into a correlation between the bestfit $K_{0}$ values and the LIS intensity at the GeV scale which, in turn, determines the absolute scale of the modulated CR flux $J_{0}$ at the $\mathrm{GeV}$ scale. The $K_{0}-J_{0}$ correlation is also discussed in Sec. IVA. To estimate the impact of the LIS uncertainty on the temporal dependence of the best-fit parameters of CR diffusion in the heliosphere, we proceeded as in Refs. [49,53]. We performed dedicated runs of fitting procedure for a large number of randomly generated LIS functions where, for each input LIS, the time series of the diffusion parameters were determined. In practice, the LIS functions were generated using the Monte Carlo framework in Ref. [48], i.e., according to the probability density function of the Galactic CR transport parameters. With this procedure, the systematic uncertainties associated with the LIS modeling are included in the final errors with a proper account for their correlations.

\section{The reversal phase}

The parameter $T_{\text {rev }}$ marks the epoch of the 2013 magnetic reversal, where the HMF flipped from negative to positive 
polarity states. The polarity of the HMF, however, is well defined only for $t \ll T_{\text {rev }}$ and $t \gg T_{\text {rev }}$, where the largescale HMF structure follows a dipole-like Parker field to a good approximation. During reversal, the polarity of the field is less sharply defined, and the HMF field follows a more complex dynamic (e.g., Ref. [58]). A way to account for this situation is to use a generalized definition of polarity, such as the BMA reconstruction $\hat{A}$ in Fig. 5 which ranges from -1 to +1 . For any given parameter configuration $q$, the flux model $J_{m}(E, \vec{q})$ can be built as a linear combination of fluxes with defined polarities, weighted by a transition function $\mathcal{P} \equiv(1-\hat{A}) / 2$ :

$$
J_{m}(E, \vec{q})=J_{m}^{-}\left(E ; \vec{q}^{-}\right) \mathcal{P}+J_{m}^{+}\left(E ; \vec{q}^{+}\right)[1-\mathcal{P}],
$$

where $\vec{q}^{( \pm)}=\left\{\alpha, B_{0}, A^{ \pm}, K_{0}, a, b\right\}$ is a vector of parameters with fixed polarity $A= \pm 1$ and $J_{m}^{( \pm)}$are the corresponding modulated fluxes. The weight $\mathcal{P}$ ranges from 1 to 0 , for floating polarity $\hat{A}$ ranging from -1 to 1 . The time dependence of the $\mathcal{P}(t)$ function associated to the polarity $\hat{A}(t)$ in Fig. 5 can be expressed as follows:

$$
\mathcal{P}(t)=\left[1+e^{\left(t-T_{\mathrm{rev}}\right) / \delta T}\right]^{-1},
$$

where $\delta T \cong 3$ months. The transition function $\mathcal{P}(t)$ is such that $\mathcal{P} \cong 0(\mathcal{P} \cong 1)$ for $t \lesssim 3 T_{\text {rev }}\left(t \gtrsim 3 T_{\text {rev }}\right)$ within $1 \%$ level of precision; i.e., when $t=T_{\text {rev }} \pm 3 \delta T$, the flux is $99 \%$ made of a fixed polarity, while the maximum mixing is for $t=T_{\text {rev }}$ when $\mathcal{P}(t)=1 / 2$. It is worth noticing that Eq. (20) relies on the implicit assumption that, during HMF reversal, the modulated flux of CRs can be regarded as a superposition of fluxes with positive and negative polarity states. We also note that this approach enabled us to define the transition epoch, from a smoothed definition of the polarity $\hat{A}$, which is indicated by the shaded area in Fig. 5. Such a definition of the transition epoch is consistent with estimations of the reversal epoch based on the dynamics of the HMF topology $[58,59]$.
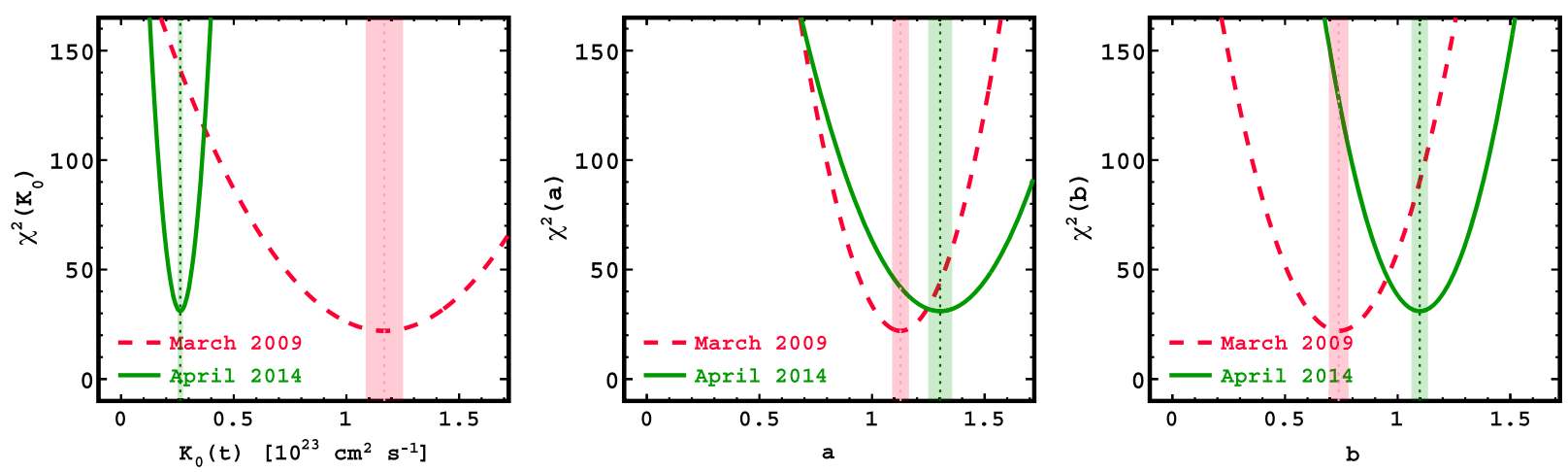

FIG. 7. One-dimensional projections of the $\chi^{2}$ surfaces as a function of the transport parameters $K_{0}, a$, and $b$ evaluated for two epochs: March 2009 (pink dashed line) and April 2014 (green continuous line). In the two epochs, representing solar minimum and solar maximum conditions, the CR flux data come from PAMELA and AMS-02, respectively. 


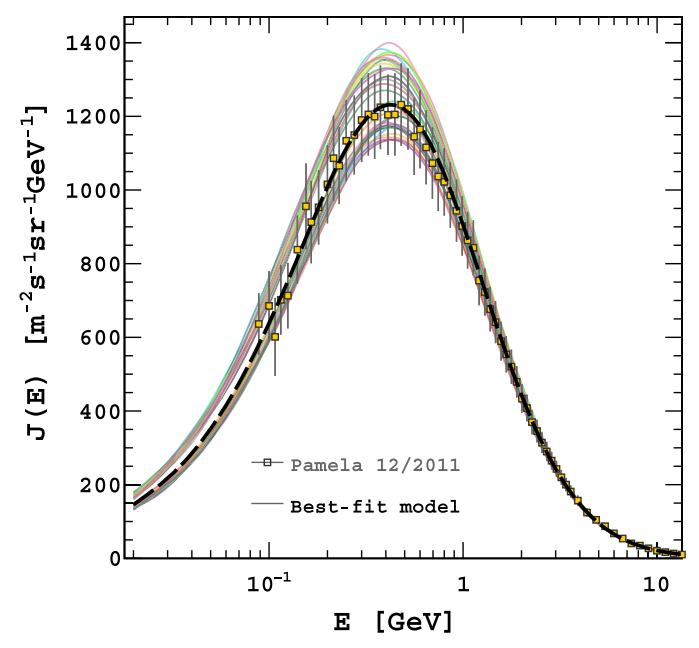

FIG. 8. Example of multilinear flux interpolation. The thick long-dashed line represents the interpolated best flux fit to the dataset for BR 2435, December 2011. The colored curves show the 32 fluxes corresponding to the array models such that $x_{i} \leq x_{\text {best }}<x_{i+1}$ (where $x=\alpha, B_{0}, K_{0}, a$, and $b$ ) used for the interpolation.

are well constrained. It can be seen that the AMS-02 data provide tight constraints on the $K_{0}$ and $b$ parameters, while the parameter $a$ is more sensitive to low-rigidity data and, thus, it is better constrained by PAMELA. After the best-fit parameters have been determined for a give set of data, the best model flux $J_{\text {best }}(E)$ is recalculated using a multilinear interpolation over the five-dimensional grid such that $x_{i} \leq x_{\text {best }}<x_{i+1}$, where $x=\alpha, B_{0}, K_{0}, a$, and $b$. In this procedure, the polarity $A$ is not involved, because it is regarded as a fixed parameter. The flux determination done under both $A^{+}$and $A^{-}$hypotheses gives the two $J^{ \pm}$fluxes of Eq. (20). The best model is shown in Fig. 8 as a thick long-dashed line, along with 32 flux calculations of all adjacent grid nodes. The model is superimposed to the data from PAMELA corresponding to December 2011 (BR 2445). During this epoch, the HMF was in well-defined negative polarity state. All fluxes in the figure are calculated for $A=-1$, i.e., with $\mathcal{P}=1$.

\section{RESULTS AND DISCUSSION}

Here, we present the results of the fitting procedure described in Sec. III C and implemented using the considered dataset on CR protons of Sec. III A. We found that the agreement between best-fit model and the measurements on the fluxes of CR protons was, in general, very good for all the datasets and over the whole rigidity range. In Fig. 9, the best-fit models for the proton fluxes are shown as colored lines for some selected epochs, along with the CR proton LIS. The calculations are compared with the data from experiments PAMELA and AMS-02 at the corresponding epochs. The long-dashed line represents the proton LIS model used in this work and presented in Sec. II C.

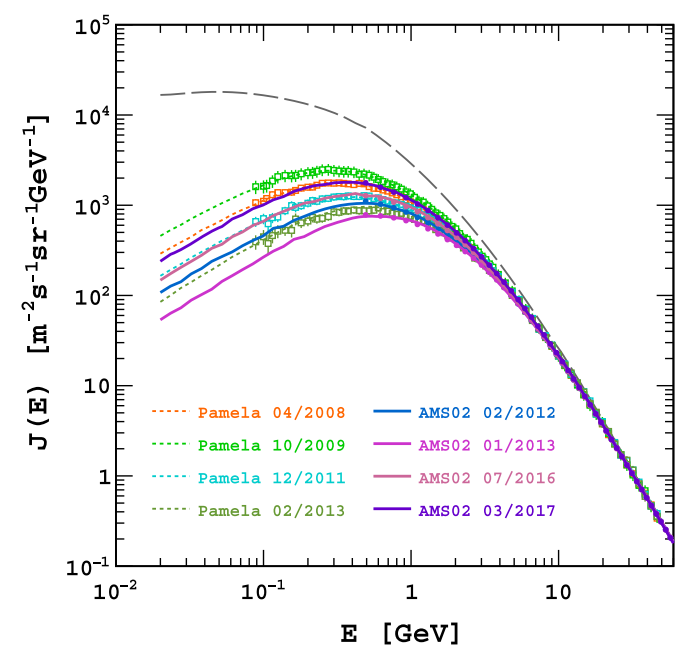

FIG. 9. Best-fit fluxes for selected datasets corresponding to PAMELA (dotted lines) and AMS-02 (solid lines) measurements. The long-dashed line represents the proton LIS used in this work.

\section{A. Temporal dependencies}

The main results on the parameter determination procedure are illustrated in Fig. 10. The figure shows the bestfit model parameters $K_{0}, a$, and $b$ as a function of the epoch corresponding to the measurements of AMS-02 (filled circles) and PAMELA (open squares). The vertical dashed line and the shaded area around it represent the reversal phase, as in the previous figures. As a proxy for solar activity, Fig. 10(d) shows the monthly SSN data. The solid line shows the smoothed SSN values, obtained with a moving average within a time window of 13 months, along with its uncertainty band. It can be seen that the diffusion parameters show a remarkable temporal dependence, and such a dependence is well correlated with solar activity. From the figure, it can be seen that the normalization of the parallel diffusion coefficient $K_{0}$ shows a clear temporal dependence. The diffusion normalization appears to be maximum in the $A<0$ epoch before reversal $\left(t \ll T_{\text {rev }}\right)$ and, in particular, during the unusually long solar minimum of 2009-2010. The minimum of $K_{0}$ is reached during solar maximum in 2014, about one year after polarity reversal. From the comparison between Figs. 10 (a) and 10(d), the $K_{0}$ parameter appears anticorrelated with the monthly SSN. Physically, larger values of $K_{0}$ imply faster CR diffusion inside the heliosphere, thereby causing a milder attenuation of the LIS, i.e., giving a higher flux of cosmic protons in the $\mathrm{GeV}$ energy region. In contrast, lower $K_{0}$ values imply slower CR diffusion, which is typical in epochs of high solar activity where the modulation effect is significant. Qualitatively, this behavior can be interpreted within the force-field approximation where, in fact, positive correlation is expected between SSN and the modulation potential $\phi \propto 1 / K_{0}$ [9]. Within the framework of the force-field model, the parameter $\phi$ is interpreted as the average kinetic energy loss of CR protons inside the heliosphere. 

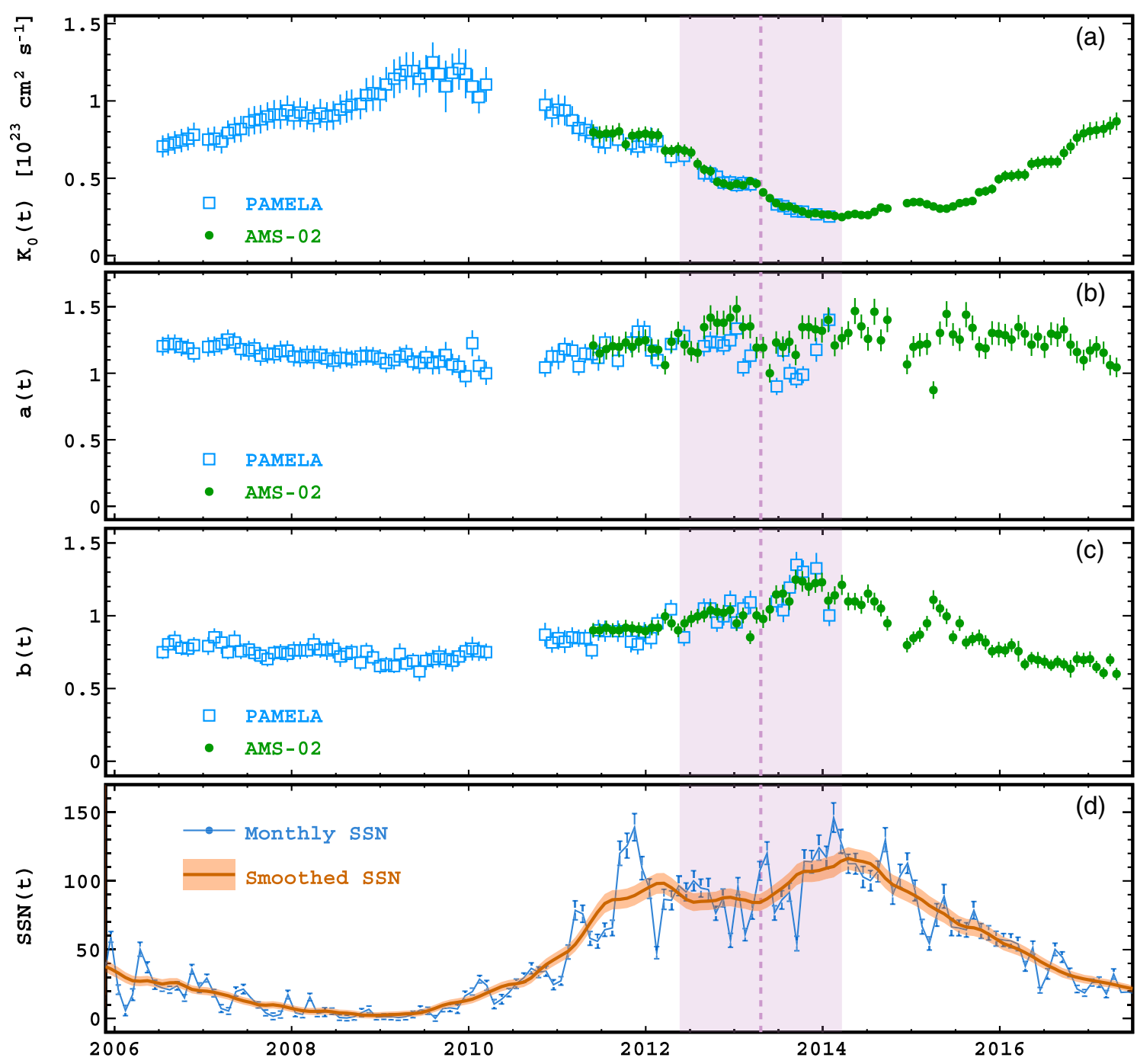

FIG. 10. Results for the best-fit model parameters $K_{0}, a$, and $b$ determined using the time-resolved proton flux measurements from PAMELA (open squared) and AMS-02 (filled circles). In (d), the monthly averaged and smoothed SSN is shown. The vertical dashed line indicates the reversal epoch $T_{\text {rev }}$, and the shaded area around it shows the transition epoch where the HMF polarity is weakly defined.

For similar reasons, a positive correlation between the bestfit $K_{0}$ value and the CR flux intensity $J_{0}$ at a given energy as can be noticed, in particular, from the comparison of Fig. 10(a) with Fig. 4. Our findings are in agreement with earlier works $[5,51,60]$. During the reversal phase, the temporal evolution of the model parameters in Fig. 10 is obtained using the weighted linear combination of model fluxes with opposite polarities given by Eq. (20). During this epoch, the diffusion of CRs is slow, and the tilt angle $\alpha$ reaches large values, typically higher than $65^{\circ}$.

The inferred $K_{0}$ values and their temporal evolution are related to the level of magnetic turbulence in the heliospheric plasma. As is clear from the figure, the diffusion is faster when the Sun is quiet with low turbulence levels and vice versa. From Eq. (8), the CR diffusion coefficients are linked to the HMF intensity and its temporal evolution, which, however, from Fig. 5, appears to be quite shallow in the epoch considered. As recently suggested in Ref. [61], the relation between the diffusion coefficient and the magnitude of the local HMF can be described by a power law, but the two quantities obey to different relationships for ascending and descending phases of the solar cycle. Physical explanation for these behaviors may involve temporal variations in the spectrum of heliospheric turbulence during the solar cycle $[62,63]$, that we discuss in the following. Investigations on the correlations between solar and diffusion parameters are made in Sec. IV C.

\section{B. The evolving turbulence}

The $a$ and $b$ parameters shown in Fig. 10 describe the rigidity dependence of CR diffusion tensor $K_{\|}$below and 
above the break value $R_{k}$. These parameter can test how the Sun variability affects the spectrum of magnetic irregularities of the heliospheric plasma, that is, its turbulence spectrum. From the figure, it can be noted that both parameters show a characteristic temporal dependence in the epoch considered. In the negative polarity epoch of $t \ll T_{\text {rev }}$ and, in particular, during solar activity minimum, the spectral indices of CR diffusion are seen to vary smoothly and slowly with time.

The two spectral indices show a different temporal dependence. The index $a$ is found to be essentially time independent, with an average value of $a=1.21 \pm 0.06$, while the index $b$ shows a distinct long-term evolution in the considered period. During the long unusual minimum from 2006 to 2009, $b$ remains constant at a value of $b=0.74 \pm 0.03$, as long as the solar activity is quiet and the corresponding number of monthly sunspots is below 250. Subsequently, in 2010-2011, when the ascending phase of the solar cycle sets in, $b$ starts to increase steadily. During this period, the CR flux decreases steadily as well. The increase keeps going during the whole reversal phase, i.e., at full maximum solar activity. Here, the $b$ parameter reaches an average maximum value of $1.3 \pm 0.07$. After this phase and during the flux recovery phase in the positive polarity epoch, the index $b$ decreases steadily during the descending phase of the solar cycle, until it recovers the values of the previous solar minimum. Instead, the index $a$ shows no prominent features over the whole descending phase.

It should be noted, however, that the $a$ parameter is poorly constrained in the $A>0$ phase, because the AMS-02 data are available only above $1 \mathrm{GV}$ of rigidity, and, thus, they are not highly sensitive to this parameter. From the figure, it can be seen that the index $b$ is negatively correlated with the diffusion normalization parameter $K_{0}$ : During minimum, where $K_{0}$ is large and the $\mathrm{CR}$ diffusion is therefore fast, its rigidity dependence is shallow $(b \approx 0.8)$ in comparison to solar maximum, where diffusion is slow and its rigidity dependence is more pronounced $(b \approx 1.3)$. Since the two indices are related to the power spectrum of the heliospheric turbulence, they could be used to infer the spectral index $\nu$ of the power spectrum density of HMF irregularities (see Sec. II B). Keeping in mind that $\lambda_{\|} \propto R^{2-\nu}$, the index $a$ is related to the power spectrum density in the energycontaining range, while the index $b$ is related to the power spectrum in the inertial range of the turbulent energy cascade of HMF. The results indicate that the diffusion spectrum in the energy-containing regime does not depend on the solar activity, while, in the inertial range, the spectrum appears to evolve as a function of the solar activity, with a clear delayed peak at the solar maximum. The spectral index of the turbulence in the energycontaining range is $\nu_{e c}=0.79 \pm 0.13$ over all the period examined in this work, while in the inertial range the spectral index evolves from $\nu_{\text {in }}=0.74 \pm 0.08$ at solar minimum to $\approx 1.3 \pm 0.15$ during the solar maximum.

The temporal and rigidity dependence of the CR mean free path $\lambda_{\|}(t, R)$ can be determined from Eq. (8) using our best-fit parameters. At the $R \approx 1 \mathrm{GV}$ rigidity scale, our $\lambda_{\|}$is found to range between 0.05 and $0.3 \mathrm{AU}$, depending on solar activity. This result is in excellent agreement with the large collection made in Ref. [64] of observational measurements on the scattering mean free path [65]. In addition, our results show that the CR variability involves the rigidity dependence of the diffusion tensor, in particular, via the spectral indices $a=a(t)$ and $b=b(t)$. An important implication of this finding is that the parallel diffusion coefficient cannot be written as a product $K_{\|}(t, R)=f(t) \times g(R)$, where a universal rigidity dependence $g(R)$ is modulated in amplitude by means of a factorized function $f(t)[49,60]$. Mathematically, this makes the $K_{\|}(t, R)$ function of Eq. (8) a nonseparable function of rigidity and time variables. Physically, it indicates that the HMF turbulence spectrum varies significantly over the solar cycle, depending on the cycle phase. In particular, the power spectrum is observed to be steeper around solar maximum and flatter during solar minimum, with a quasiperiodical pattern. The temporal variability of HMF turbulence is also studied from the analysis of neutron monitor data [62]. These findings suggest that, during epochs of quiet activity, kinetic self-organized turbulence dominates the $\mathrm{CR}$ spectrum, such as, e.g., a Kolmogorov-type cascade, while random processes and transient events in the heliosphere play a key role during high-activity epochs of the solar cycle. The use of wider sets of data may allow one to provide better clarification on such a behavior.

\section{Cross-correlations}

We now inspect the running cross-correlation between solar and transport parameters. Figure 11 displays the scatter diagrams of the best-fit diffusion parameters against the BMA reconstruction of the local HMF value $\hat{B}_{0}$ (left column) and the HCS tilt angle $\alpha$ (right column). In Fig. 11(a), the diffusion normalization parameter $K_{0}$ is shown. The different markers are used to indicate the reconstructions obtained during epochs of positive (blue circles) and negative polarity (pink squares), as well as during reversal phase (green triangles). This behavior can be compared with the one found by Wang et al. [61] where, from an analysis of the ascending and descending phases of the solar cycle (both during negative polarity), two distinct power-law relations were observed between the diffusion coefficient and local HMF magnitude. Our results confirm that the relationship between $K_{0}$ and $B_{0}$ becomes complex when the examination is done over a large fraction of the solar cycle that includes polarity changes. In particular, two distinct relationships can be observed for $A<0$ and $A>0$ 

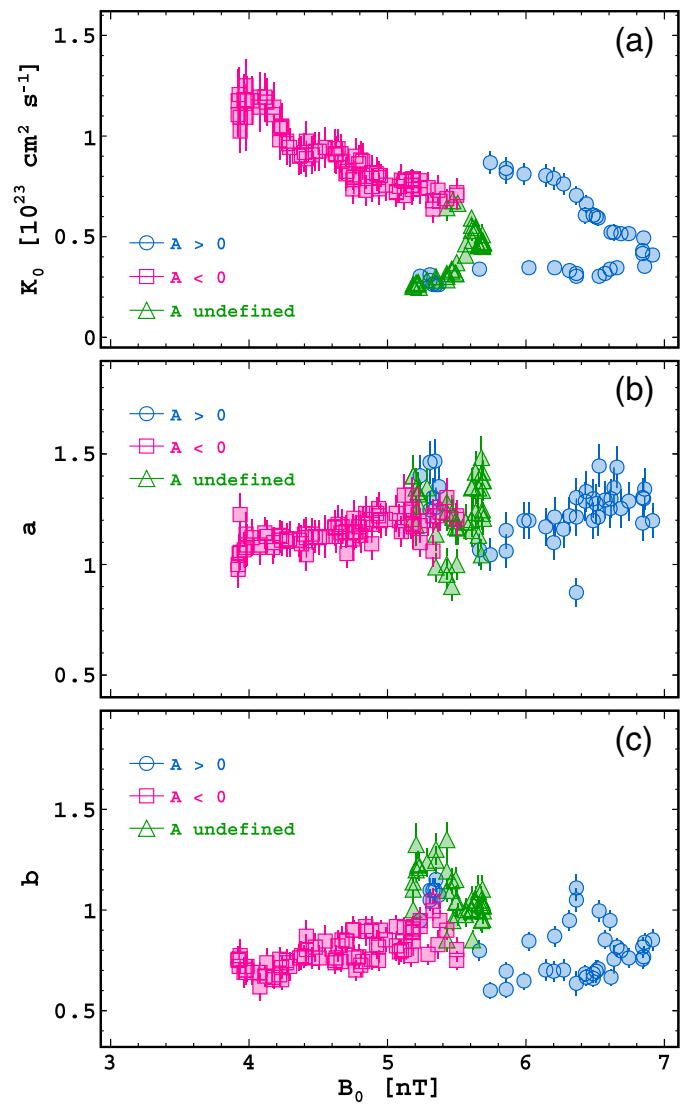

FIG. 11. Scatter plots showing the correlation between the bestfit diffusion parameters and BMA reconstruction of the local HMF $\hat{B}_{0}$. The results are divided in groups of positive polarity (blue circles), negative polarity (pink squares), and reversal phase (green triangles).

polarity conditions. Regarding the correlation between the spectral index parameters $a$ and $b$ with the HMF magnitude $\hat{B}_{0}$, smoother relationships were found. The index $a$ is nearly constant with time, while the index $b$ increases slowly during solar maximum, i.e., during the reversal phase. Both parameters are seen to depend only weakly on the polarity phase, and no particular cross-correlation is observed between two spectral indices. The scatter plot of $K_{0}$ versus tilt angle is also shown, in Fig. 12, where, again, the different styles of the markers refer to the different phases of solar activity. The dependence is similar to that observed with the HMF intensity, showing a pronounced negative correlation and a characteristic modulation loop.

The correlation between the flux intensity $J_{0}$ and the diffusion normalization $K_{0}$ is shown in Fig. 13. In this figure, the flux intensity $J_{0}$ is extracted from the data at the reference kinetic energy $E_{0}=0.49-0.62 \mathrm{GeV}$, as in Fig. 4, while $K_{0}$ is the best-fit value at the corresponding epoch. From the figure, the CR flux intensity appears, in general, well correlated to the normalization factor of the diffusion coefficient, which appears to be the driving parameter of the modulation model. It can also be seen that relationship

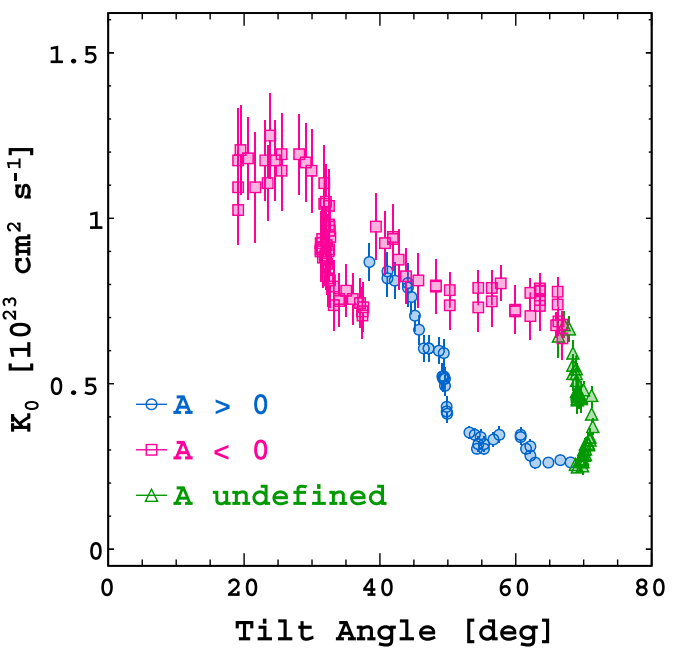

FIG. 12. Scatter plot of the best-fit parameter $K_{0}$ versus the HCS tilt angle. The results are divided in groups of positive polarity (blue circles), negative polarity (pink squares), and reversal phase (green triangles).

between $J_{0}$ and $K_{0}$ is remarkably linear during epochs of well-defined polarity. We describe it with the following empirical relation:

$$
J_{0}\left(K_{0}\right)=\eta K_{0}+J_{\text {off }}
$$

By making separate fits for the two polarity epochs, we obtained $\eta^{+}=(2212 \pm 250) \times 10^{-23}$ for $A>0$ and $\eta^{-}=(1929 \pm 260) \times 10^{-23} \mathrm{~cm}^{-4} \mathrm{GeV}^{-1} \mathrm{sr}^{-1}$ for $A<0$. The best-fit offsets are $J_{\text {off }}^{+}=-46 \pm 21$ for positive polarity and $J_{\text {off }}^{-}=-286 \pm 68 \mathrm{~m}^{-2} \mathrm{~s}^{-1} \mathrm{GeV}^{-1} \mathrm{sr}^{-1}$ for negative

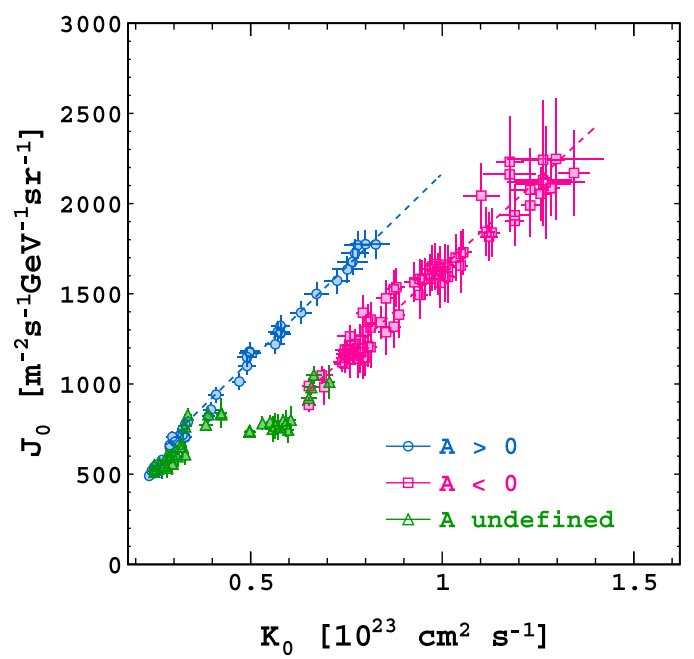

FIG. 13. Scatter plot of the CR flux $J_{0}$, evaluated at the reference energy interval $E_{0}=0.49-0.62 \mathrm{GeV}$, against the normalization factor of the diffusion tensor $K_{0}$. The color coding is the same as in Fig. 12. During the phases of well-defined polarity, $J_{0}$ shows a distinct dependence on the diffusion strength parameter that has been fit with Eq. (22) (dashed lines). 
polarity. The two fits are shown in Fig. 13 as dashed lines. It is interesting to note that, within the fitting errors, the two slopes $\eta^{+}$and $\eta^{-}$turned out to be consistent with each other; i.e., the slope of $J_{0}\left(K_{0}\right)$ is polarity and charge-sign independent. The polarity effect results in different offsets $J_{\text {off }}^{ \pm}$for the two phases. This result may help to quantify the effects of drift motions to the CR modulation. The diffusion coefficient appears to be independent of the $\hat{q} A$ sign product, as indicated by the consistency between $\eta^{+}$and $\eta^{-}$values from the fit. For a given $K_{0}$ value, the resulting difference in the fluxes is only due to the opposite directions of the net drift and convective flux for epochs of opposite polarities. The quantity $\Delta J \equiv J_{\text {off }}^{+}-J_{\text {off }}^{-}$can be used as a measurement of the net effect of drift on the total CR flux, for a given level of CR diffusion.

We also note that, in the figure, the fit results obtained under periods of undefined polarity (green triangles) connect smoothly the two regimes. In this epoch, the role of drift is not well understood, but the flux $J_{0}$ remains correlated with $K_{0}$. To close the loop, it may take an entire cycle of magnetic polarity.

\section{Lags and loops}

From Fig. 10, it can be noticed that a time shift of a few months is present between the smoothed SSN [the $S(t)$ function] and the best-fit modulation parameters $K_{0}(t), a(t)$, and $b(t)$. For instance, the highest CR flux intensity was reached around October 2009, with $J^{\max }=2289 \pm 220 \mathrm{~m}^{-2} \mathrm{~s}^{-1} \mathrm{GeV}^{-1} \mathrm{sr}^{-1}$, i.e., about eight months after the SSN minimum of February 2009. Similarly, the minimum flux intensity was observed around February 2014, $J^{\mathrm{min}}=498 \pm 23 \mathrm{~m}^{-2} \mathrm{~s}^{-1} \mathrm{GeV}^{-1} \mathrm{sr}^{-1}$, while solar maximum occurred in April 2013. To estimate the average time lag between $K_{0}(t)$ and the smoothed SSN $S(t)$, we compare the correlation between $K_{0}(t)$ and $S\left(t-\Delta T_{\text {lag }}\right)$. The best value for the lag $\Delta T_{\text {lag }}$ can be obtained by a scan of $\Delta T_{\mathrm{lag}}$, in order to determine the Pearson linear correlation coefficient $\rho$ as a function of $\Delta T_{\text {lag }}$. The $\Delta T_{\text {lag }}$ parameter which maximizes $\rho$ is then taken as the best estimate of the average time lag between the SSN and CR modulation parameters. For the analyzed period, we obtain $\Delta T_{\text {lag }}=11.4 \pm 1.4$ months. Thus, on average, the modulation of CRs observed at the epoch $t$ is related to manifestations of solar activity at the epoch $t-\Delta T_{\text {lag. }}$. The correlation between diffusion parameters and smoothed SSN is shown in Fig. 14, where the model parameters at the epoch $t$ are shown as a function of the SSN at the same epoch (left column) and at the epoch $t-\Delta T_{\text {lag }}$ (right column). In general, when the time lag is not taken into account, the diffusion normalization $K_{0}(t)$
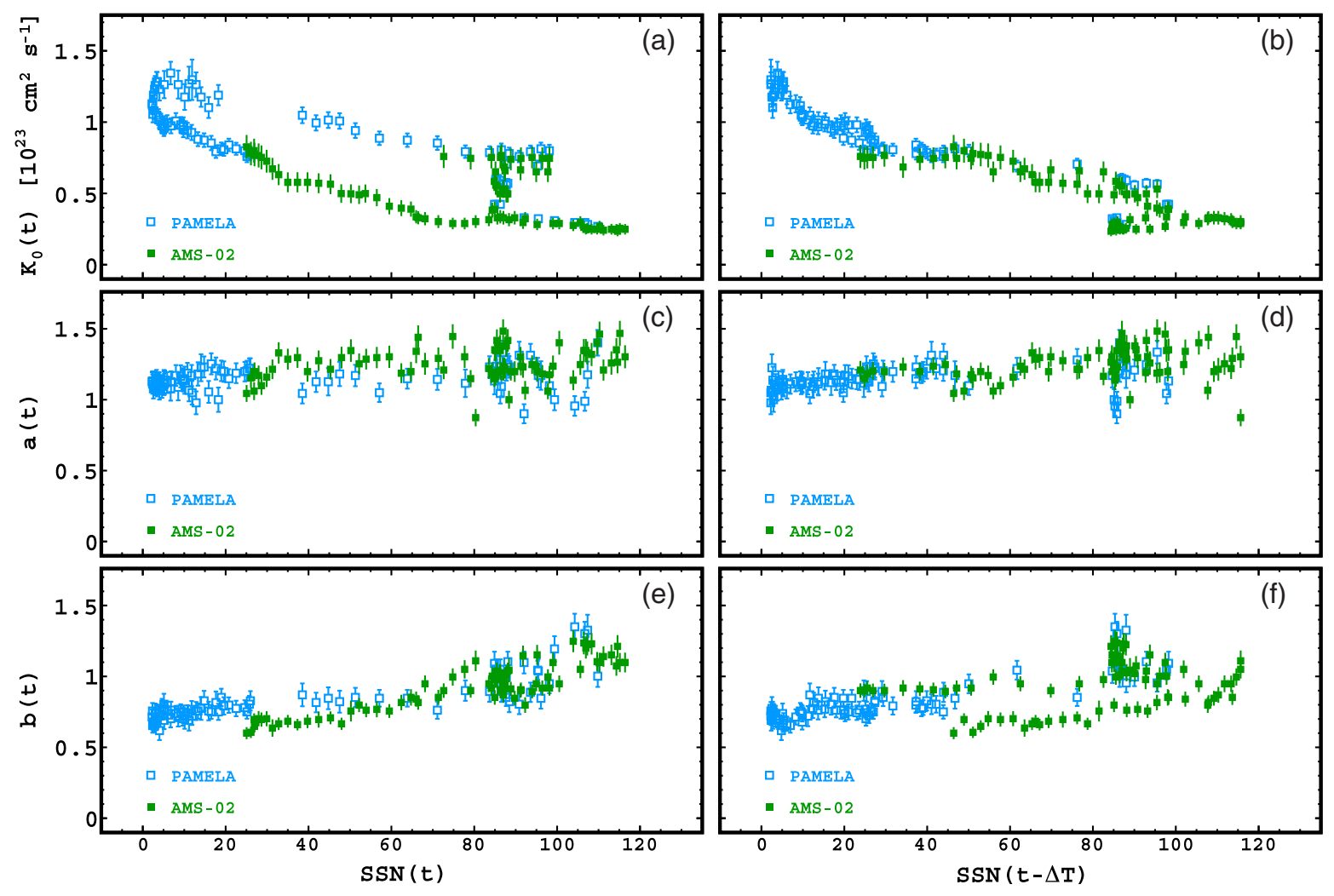

FIG. 14. Model parameters as a function of the SSN. The left column displays the parameters as a function of SSN $(t)$ versus the SSN at the same epoch, and the right column displays the parameters as a function of $\operatorname{SSN}\left(t-\Delta T_{\text {lag }}\right)$. The AMS-02 and PAMELA data are represented by the red and black dots, respectively. 
appears as a multivalued function of SSN, showing a characteristic hysteresis structure over the different phases of the solar cycle. When the lag is taken into account, the curve of $K_{0}$ versus SSN shrinks, approaching a singlevalued function. This would allow one, in principle, to forecast the modulation parameters at the epoch $t$ from observations of SSN made in advance by $\Delta T_{\text {lag. }}$. However, the $a$ and $b$ parameters versus the delayed SSN do not show clear one-to-one relationships, which suggests that the use of a single lag value may be a too simplistic approach. The calculated lag depends weakly on the BMA averages used to define the heliosphere status. On the other hand, the BMA procedure in Sec. III B is well motivated by the observation of such a lag. In this respect, an estimate of the uncertainty on $\Delta T_{\text {lag }}$ can be done by varying the time window $T_{\mathrm{BMA}}$ used to get the average conditions $\left(B_{0}\right.$ and $\alpha$ ) of the heliosphere. Our estimation of $\Delta T_{\text {lag }}$ is fairly consistent with other recent works $[3,51,66]$. Nonetheless, there are some discrepancies with the reported values if one accounts for even or odd cycle dependence of the lag. Our estimation of the time lag lies in solar cycle 24, but it appears longer than that reported in previous evennumbered solar cycles, though it is comparable to the lag observed in odd-numbered solar cycles [67-69]. In this respect, as well as in other characteristics, cycle 24 is unusual when compared to previous even cycles. Other differences may be related to the rigidity of CR particles, as past studies are based on neutron monitor rates. The global dependence of the time lag upon the solar cycle and on the rigidity of the CR particles will be addressed in a forthcoming paper.

\section{CONCLUSIONS AND DISCUSSION}

Thanks to the recent availability of time-resolved data from space, the study of CRs in the heliosphere has become an active topic of investigation. In particular, the recent data released by AMS-02 and PAMELA on the monthly evolution of proton and helium permits new investigation of the solar modulation phenomenon over a large fraction of the solar cycle. These data have triggered new efforts at establishing advanced models of CR propagation in the heliosphere [22,70-73]. In particular, many recent studies were focused on specific aspects of the $\mathrm{CR}$ modulation such as, e.g., the particle dependence of CR diffusion [5,49], the relationship between modulation and solar activity proxies $[61,74]$, the derivation of improved LIS evaluation [20,73], or the extraction of CR modulation parameters using statistical inference [5], which is also the main goal of the present paper. More specifically, in this paper, we have investigated the propagation of Galactic CRs in the heliosphere using a numerical model based on stochastic simulations and calibrated by means of a large set of experimental data. The data consist of time series of CR proton fluxes reported by AMS-02 and PAMELA experiments in low Earth orbit. The measurements are made on a 27-day basis, corresponding to a solar rotational period, and cover a time range of 11 years, corresponding to a solar cycle period. The sample include epochs of very different solar conditions such as solar minimum, solar maximum, and ascending and descending phases, as well as positive and negative HMF polarity states. The time range and resolution of these data is, therefore, optimal for the study of long-term modulation of Galactic CRs and, in particular, for investigating the influence of solar variability in the diffusive propagation of CRs in the heliospheric turbulence.

In our calculations, we have used, as time-dependent physical inputs, BMA values of the tilt angles $\alpha$ of the HCS, the local HMF strength at $1 \mathrm{AU} B_{0}$, and the magnetic polarity $A$. These quantities constitute very good proxies for solar activity. In this analysis, we have been focused on the parameters describing the temporal and rigidity dependence of CR diffusion. We have determined the time series of the diffusion normalization, $K_{0}$, and that of the spectral indices $a$ and $b$ that control the dependence of CR diffusion upon rigidity.

In practice, to perform a statistical inference using the data and to account for the evolving conditions of the heliospheric plasma, we have built a large array of differential energy fluxes $J(E)$, evaluated at Earth's location, corresponding to 938400 parameter configurations. To sample such a six-dimensional parameter space, we have simulated about 14 billion trajectories of cosmic protons in the interplanetary space. Each simulated particle was backwardly propagated from Earth's vicinity to the heliospheric boundaries. The array of models generated in this work can be used to estimate the modulation parameters of CR protons at any epoch and for any set of experimental data, ranging from $20 \mathrm{MeV}$ to hundreds of $\mathrm{GeV}$ of kinetic energy. We also note that, in our model, the time dependence of the problem is treated by providing a time series of steady-state solutions for $J_{\mathrm{p}}$ associated with a time series of input parameters $k_{0}$, which is a simplification. Such an approach stands as long as the timescales between CR transport in the heliosphere do not exceed the analyzed changes in solar activity. To extend the analysis to a smaller timescale (e.g., daily) or to lower energies (e.g., MeVscale), a time-dependent solution of the Parker equation should be considered. Nonetheless, we also stress that the time series of best-fit parameters derived in this work should be regarded as effective values, averaged over the $\mathrm{CR}$ propagation histories, not necessarily representing the instantaneous conditions of the heliospheric plasma.

Our approach is also simplified in several aspects, for example, regarding the rigidity and spatial dependence of the diffusion tensor or its perpendicular components. Nonetheless, in comparison to our earlier works, we have introduced several new recipes that capture most of the relevant features of $\mathrm{CR}$ propagation in the heliosphere. 
The agreement of our calculations with the CR flux data is very satisfactory. As we have shown, using CR proton data, it is possible to determine the detailed evolution of the rigidity dependence of the diffusion coefficient with the solar activity and, thus, the physical nature of the turbulence embedded in the frozen-in HMF carried out by the SW. Our findings indicate that solar variability has an important effect on the turbulence spectrum of HMF irregularities, and an imprint of this mechanism can be observed in the rigidity dependence of the diffusion tensor. In particular, we have reported a remarkable long-term dependence for the two spectral indices $a$ and $b$. These results show that the turbulence regime evolves with time, following the solar cycle, and, thus, the temporal and rigidity dependencies of $\mathrm{CR}$ diffusion coefficients cannot be described by a separable function of the type $K_{\|}(t, R) \equiv K_{0}(t) \times f(R)$. In this respect, we remark that the time-rigidity separability for CR diffusion is assumed by several models of solar modulation, although such an assumption is not supported by theoretical considerations $[19,60,61]$. Moreover, the study of the correlation between solar and diffusion parameters reveals charge-signdependent features in the CR modulation effect, such as different patterns for the different phase of the HMF polarity cycle.

We remark that solar cycle 24 has been unusual when compared to the previous cycles; therefore, also the CR modulation conditions were unusual. The solar minimum between cycles 23 and 24 was quite longer and deeper than expected $[32,67]$, while the maximum of cycle 24 was the smallest recorded in a century of standardized SSN observations and with a double-peak structure [75]. In our analyzed data sample, the correlation between CR flux modulation and solar activity as measured by the SSN is apparent. The CR proton intensity modulation, in antiphase with solar activity, in the considered period shows an average time lag of about 11 months. A next phase of this work is to study the dependence of the lag on solar activity parameters (such as SW speed or HMF polarity) and CR transport properties (such as diffusion or drift coefficients), in order to understand the dynamics of the physical mechanisms behind the solar modulation phenomenon. Further steps also include the implementation of a better description of the HMF, the diffusion tensor, and the drift reduction factor during solar maximum. In particular, we assumed "full drift" at any phase of the cycle, including the HMF reversal epoch, where the modulated flux of CRs was modeled as a superposition of fluxes with positive and negative polarity states. While our approach provided a good description of the flux evolution in the reversal region, one may argue that large-scale drift may be suppressed during solar maximum due to the more chaotic structure of the HMF. This idea can, in principle, be tested using the data. In particular, the availability of time-dependent measurements on $\mathrm{CR}$ antiprotons will be precious to study the modulation effect across solar maximum. Data of the temporal dependence of CR antiprotons are still lacking, but the AMS-02 experiment has the capability of making such a measurement.

\section{ACKNOWLEDGMENTS}

We acknowledge the support of Italian Space Agency (ASI) under Agreement No. ASI-UniPG 2019-2-HH.0. B. K. acknowledge support from Agreement No. ASIINFN 2014-037-R.1-2017 and M. G. and F. D. from No. ASI-INFN 2019-19-HH.0. The cosmic ray data used in this work have been retrieved through the cosmic ray database of the ASI Space Science Data Center. Data on the Sun's polar magnetic field and tilt angle are taken from the Wilcox Solar Observatory at Stanford University. Interplanetary HMF data of the Advanced Composition Explorer have been downloaded from the OMNIWeb service of the NASA Space Physics Data Facility. Data on the sunspot numbers are provided by the Solar Influence Data Analysis Center at the Royal Observatory of Belgium, Brussels.
[1] M. S. Potgieter, Solar modulation of cosmic rays, Living Rev. Solar Phys. 10, 3 (2013).

[2] I. G. Usoskin, H. Kananen, K. Mursula, P. Tanskanen, and G. A. Kovaltsov, Correlative study of solar activity and cosmic ray intensity, J. Geophys. Res. 103, 9567 (1998).

[3] E. Ross and W. Chaplin, The behaviour of galactic cosmicray intensity during solar activity cycle 24 , Sol. Phys. 294, 8 (2019).

[4] J. T. Hoeksema, The large-scale structure of the heliospheric current sheet during the ULYSSES epoch, Space Sci. Rev. 72, 137 (1995).
[5] C. Corti, M. S. Potgieter, V. Bindi, C. Consolandi, C. Light, M. Palermo, and A. Popkow, Numerical modeling of galactic cosmic ray proton and helium observed by AMS-02 during the solar maximum of solar cycle 24, Astrophys. J. 871, 253 (2019).

[6] M. J. Boschini, S. Della Torre, M. Gervasi et al., Solution of heliospheric propagation: Unveiling the local interstellar spectra of cosmic-ray species, Astrophys. J. 840, 115 (2017).

[7] P. Bobik, M. J. Boschini, S. Della Torre et al., On the forward-backward-in-time approach for Monte Carlo 
solution of Parker's transport equation: One-dimensional case, J. Geophys. Res. 121, 3920 (2016).

[8] M.S. Potgieter, The global modulation of cosmic rays during a quiet heliosphere: A modeling perspective, Adv. Space Res. 60, 848 (2017).

[9] N. Tomassetti, Solar and nuclear physics uncertainties in cosmic ray propagation, Phys. Rev. D 96, 103005 (2017).

[10] J. W. Norbury, K. Whitman, K. Lee, T. C. Slaba, and F. F. Badavi, Comparison of space radiation GCR models to recent AMS data, Life Sci. Space Res. 18, 64 (2018).

[11] A. I. Mrigakshi, D. Matthiä, T. Berger, G. Reitz, and R. F. Wimmer-Schweingruber, Assessment of galactic cosmic ray models, J. Geophys. Res. 117, A08109 (2012).

[12] F. A. Cucinotta, M. Alp, B. Rowedder, and M. Y. Kim, Safe days in space with acceptable uncertainty from space radiation exposure, Life Sci. Space Res. 5, 31 (2015).

[13] M. Aguilar, L. A. Cavasonza, B. Alpat et al., Observation of Fine Time Structures in the Cosmic Proton and Helium Fluxes with the Alpha Magnetic Spectrometer on the International Space Station, Phys. Rev. Lett. 121, 051101 (2018).

[14] M. Aguilar, L. A. Cavasonza, B. Alpat et al., Observation of Complex Time Structures in the Cosmic-Ray Electron and Positron Fluxes with the Alpha Magnetic Spectrometer on the International Space Station, Phys. Rev. Lett. 121, 051102 (2018).

[15] O. Adriani, G. C. Barbarino, G. A. Bazilevskaya et al., Time dependence of the proton flux measured by PAMELA during the 2006 July-2009 December solar minimum, Astrophys. J. 765, 91 (2013).

[16] M. Martucci, R. Munini, M. Boezio et al., Proton fluxes measured by the PAMELA experiment from the minimum to the maximum solar activity for solar cycle 24, Astrophys. J. 854, L2 (2018).

[17] A. C. Cummings, E. C. Stone, B. C. Heikkila, N. Lal, W. R. Webber, G. Jóhannesson, I. V. Moskalenko, E. Orlando, and T. A. Porter, Galactic cosmic rays in the local interstellar medium: Voyager 1 observations and model results, Astrophys. J. 831, 18 (2016).

[18] E. N. Parker, The passage of energetic charged particles through interplanetary space, Planet. Space Sci. 13, 9 (1965).

[19] H. Moraal, Cosmic-ray modulation equations, Space Sci. Rev. 176, 299 (2013).

[20] C. R. Zhu, Q. Yuan, and D. M. Wei, Studies on cosmic-ray nuclei with Voyager, ACE, and AMS-02. I. Local interstellar spectra and solar modulation, Astrophys. J. 863, 119 (2018).

[21] R. Kappl, SolarProp: Charge-sign dependent solar modulation for everyone, Comput. Phys. Commun. 207, 386 (2016).

[22] M. J. Boschini, S. Della Torre, M. Gervasi, G. La Vacca, and P. G. Rancoita, Propagation of cosmic rays in heliosphere: The HELMOD model, Adv. Space Res. 62, 2859 (2018).

[23] R. D. Strauss and R. Effenberger, A hitch-hiker's guide to stochastic differential equations: Solution methods for energetic particle transport in space physics and astrophysics, Space Sci. Rev. 212, 151 (2017).

[24] A. Kopp, I. Büsching, R. D. Strauss, and M. S. Potgieter, A stochastic differential equation code for multidimensional
FokkerPlanck type problems, Comput. Phys. Commun. 183, 530 (2012).

[25] S. E. S. Ferreira and M. S. Potgieter, Long-term cosmic-ray modulation in the heliosphere, Astrophys. J. 603, 744 (2004).

[26] E. N. Parker, Dynamics of the interplanetary gas and magnetic fields, Astrophys. J. 128, 664 (1958).

[27] J. R. Jokipii and B. Thomas, Effects of drift on the transport of cosmic rays. IV: Modulation by a wavy interplanetary current sheet, Astrophys. J. 243, 1115 (1981).

[28] J. R. Jokipii and J. Kota, The polar heliospheric magnetic field, J. Geophys. Res. 16, 1 (1989).

[29] H. Fichtner, S. R. Sreenivasan, and H. J. Fahr, Cosmic ray modulation and a non-spherical heliospheric shock, Astron. Astrophys. 308, 248 (1996), https://ui.adsabs.harvard.edu/ abs/1996A\%26A...308..248F/abstract.

[30] B. Heber and M.S. Potgieter, Cosmic rays at high heliolatitudes, Space Sci. Rev. 127, 117 (2006).

[31] A. C. Cummings and E. C. Stone, Characteristics of the termination shock: Insights from Voyager, AIP Conf. Proc. 781, 273 (2005).

[32] M. S. Potgieter, E. E. Vos, M. Boezio, N. De Simone, V. Di Felice, and V. Formato, Modulation of galactic protons in the heliosphere during the unusual solar minimum of 2006 to 2009, Sol. Phys. 289, 391 (2014).

[33] J. R. Jokipii, Cosmic-ray propagation. I. Charged particles in a random magnetic field, Astrophys. J. 146, 480 (1966).

[34] J. Giacalone and J. R. Jokipii, The transport of cosmic rRays across a turbulent magnetic field, Astrophys. J. 520, 204 (1999).

[35] W. H. Matthaeus, G. Qin, J. W. Bieber, and G. P. Zank, Nonlinear collisionless perpendicular diffusion of charged particles, Astrophys. J. 590, L53 (2003).

[36] A. Shalchi, J. W. Bieber, W. H. Matthaeus, and G. Qin, Nonlinear parallel and perpendicular diffusion of charged cosmic rays in weak turbulence, Astrophys. J. 616, 617 (2004).

[37] A. Shalchi, Perpendicular transport of energetic particles in magnetic turbulence, Space Sci. Rev. 216, 23 (2020).

[38] K. H. Kiyani, K. T. Osman, and S. C. Chapman, Dissipation and heating in solar wind turbulence: From the macro to the micro and back again, Phil. Trans. R. Soc. A 373, 20140155 (2015).

[39] R. Bruno, D. Telloni, D. DeIure, and E. Pietropaolo, Solar wind magnetic field background spectrum from fluid to kinetic scales, Mon. Not. R. Astron. Soc. 472, 1052 (2017).

[40] M. S. Potgieter, J. A. Le Roux, and R. A. Burger, Interplanetary cosmic ray radial gradients with steady state modulation models, J. Geophys. Res. 94, 2323 (1989).

[41] G. Qin, W. H. Matthaeus, and J. W. Bieber, Subdiffusive transport of charged particles perpendicular to the large scale magnetic field, Geophys. Res. Lett. 29, 1048 (2002).

[42] M. Hussein, R. C. Tautz, and A. Shalchi, The influence of different turbulence models on the diffusion coefficients of energetic particles, J. Geophys. Res. Space Phys. 120, 4095 (2015).

[43] W. R. Webber, B. Heber, and J.A. Lockwood, Time variations of cosmic ray electrons and nuclei between 1978 and 2004: Evidence for charge-dependent modulation 
organized by changes in solar magnetic polarity and current sheet tilt, J. Geophys. Res. 110, A12107 (2005).

[44] R. A. Burger and M. Hattingh, Steady-state drift-dominated modulation models for galactic cosmic rays, Astrophys. Space Sci. 230, 375 (1995).

[45] R. C. Tautz and A. Shalchi, Drift coefficients of charged particles in turbulent magnetic fields, Astrophys. J. 744, 125 (2012).

[46] N. E. Engelbrecht, R. D. Strauss, J. A. le Roux, and R. A. Burger, Toward a greater understanding of the reduction of drift coefficients in the presence of turbulence, Astrophys. J. 841, 107 (2017).

[47] N. Tomassetti, Cosmic-ray protons, nuclei, electrons, and antiparticles under a two-halo scenario of diffusive propagation, Phys. Rev. D 92, 081301(R) (2015).

[48] J. Feng, N. Tomassetti, and A. Oliva, Bayesian analysis of spatial-dependent cosmic-ray propagation: Astrophysical background of antiprotons and positrons, Phys. Rev. D 94, 123007 (2016).

[49] N. Tomassetti, F. Barão, B. Bertucci, E. Fiandrini, J. L. Figueiredo, J. B. Lousada, and M. Orcinha, Testing Diffusion of Cosmic Rays in the Heliosphere with Proton and Helium Data from AMS, Phys. Rev. Lett. 121, 251104 (2018).

[50] C. Corti, V. Bindi, C. Consolandi, and K. Whitman, Solar modulation of the local interstellar spectrum with Voyager 1, AMS-02, PAMELA, and BESS, Astrophys. J. 829, 8 (2016).

[51] N. Tomassetti, M. Orcinha, B. Bertucci, and F. Barão, Evidence for a time lag in solar modulation of galactic cosmic rays, Astrophys. J. 849, L32 (2017).

[52] N. Tomassetti, Origin of the proton-to-helium ratio anomaly in cosmic rays, Astrophys. J. 815, L1 (2015).

[53] N. Tomassetti, F. Barão, B. Bertucci, E. Fiandrini, and M. Orcinha, Numerical modeling of cosmic-ray transport in the heliosphere and interpretation of the proton-to-helium ratio in solar cycle 24, Adv. Space Res. 64, 2477 (2019).

[54] V. Di Felice, C. Pizzolotto, D. D’Urso, S. Dari, D. Navarra, R. Primavera, and B. Bertucci, Looking for cosmic ray data? The ASI cosmic ray database, Proc. Sci. ICRC2017 (2017) 1073.; see also https://tools.asdc.asi.it/CosmicRays.

[55] C. W. Smith, H. L'Heureux, N. F. Ness, M. H. Acua, L. F. Burlaga, and J. Scheifele, The ACE magnetic fields experiment, Space Sci. Rev. 86, 613 (1998).

[56] D. S. Porta, Cross correlation and time-lag between cosmic ray intensity and solar activity during solar cycles 21, 22 and 23, Astrophys. Space Sci. 363, 137 (2018).

[57] J. Minnie, J. W. Bieber, W. H. Matthaeus, and R. A. Burger, Suppression of particle drifts by turbulence, Astrophys. J. 670, 1149 (2007).

[58] X. Sun, J. T. Hoeksema, Y. Liu, and J. Zhao, On polar magnetic field reversal and surface flux transport during solar cycle 24, Astrophys. J. 798, 114 (2015).

[59] M. I. Pishkalo, On polar magnetic field reversal in solar cycles 21, 22, 23, and 24, Sol. Phys. 294, 137 (2019).
[60] R. Manuel, S. E. S. Ferreira, and M. S. Potgieter, Timedependent modulation of cosmic rays in the heliosphere, Sol. Phys. 289, 2207 (2014).

[61] B. B. Wang, X. J. Bi, K. Fang, S. J. Lin, and P. F. Yin, Timedependent solar modulation of cosmic rays from solar minimum to solar maximum, Phys. Rev. D 100, 063006 (2019).

[62] P. Vaisanen, I. Usoskin, and K. Mursula, Long-term and solar cycle variation of galactic cosmic rays: Evidence for variable heliospheric turbulence, J. Geophys. Res. Space Phys. 124, 804 (2019).

[63] L. L. Zhao, L. Adhikari, G. P. Zank, Q. Hu, and X. S. Feng, Influence of the solar cycle on turbulence properties and cosmic-ray diffusion, Astrophys. J. 856, 94 (2018).

[64] I. D. Palmer, Transport coefficients of low-energy cosmic rays in interplanetary space, Rev. Geophys. Space Phys. 20, 335 (1982).

[65] R. C. Tautz and A. Shalchi, Simulated energetic particle transport in the interplanetary space: The Palmer consensus revisited, J. Geophys. Res. 118, 642 (2013).

[66] P. Chowdhury and K. Kudela, Quasi-periodicities in cosmic rays and time lag with the solar activity at a middle latitude neutron monitor: 19822017, Astrophys. Space Sci. 363, 250 (2018).

[67] O. P. M. Aslam and Badruddin, Study of cosmic-ray modulation during the recent unusual minimum and mini-maximum of solar cycle 24, Sol. Phys. 290, 2333 (2015).

[68] M. Singh, Y. P. Singh, and Badruddin, Solar modulation of galactic cosmic rays during the last five solar cycles, J. Atmos. Terr. Phys. 70, 169 (2008).

[69] K. Iskra, M. Siluszyk, M. Alania, and W. Wozniak, Experimental investigation of the delay time in galactic cosmic ray flux in different epochs of solar magnetic cycles: 1959 2014, Sol. Phys. 294, 115 (2019).

[70] X. Luo, M. S. Potgieter, V. Bindi, M. Zhang, and X. Feng, A numerical study of cosmic proton modulation using AMS-02 observations, Astrophys. J. 878, 6 (2019).

[71] M. D. Ngobeni, O. P. M. Aslam, D. Bisschoff et al., The 3D numerical modeling of the solar modulation of galactic protons and helium nuclei related to observations by PAMELA between 2006 and 2009, Adv. Space Res. 365, 182 (2020).

[72] P. Bobik, M. Putis, Y. L. Kolesnyk, and B. A. Shakhov, Estimation of the modulation level of cosmic rays at high energies, Mon. Not. R. Astron. Soc. 503, 3386 (2021).

[73] M. J. Boschini, S. Della Torre, Gervasi et al., Inference of the local interstellar spectra of cosmic-ray nuclei $\mathrm{Z} \leq 28$ with the GalProp-HelMod framework, Astrophys. J. Suppl. Ser. 250, 27 (2020).

[74] B. B. Wang, X. J. Bi, K. Fang, S. Lin, and P. F. Yin, Solar modulation of cosmic proton and helium with AMS-02, arXiv:2011.12531.

[75] F. Clette and L. Lefèvre, The new sunspot number: Assembling all corrections, Sol. Phys. 291, 2629 (2016); see also http://www.sidc.be. 